\title{
Application of Jetstream to a Suite of Aerodynamic Shape Optimization PROBLEMS
}

by

Karla Telidetzki

A thesis submitted in conformity with the requirements

for the degree of Master of Applied Science

Graduate Department of Aerospace Engineering

University of Toronto

(C) Copyright 2014 by Karla Telidetzki 


\begin{abstract}
Application of Jetstream to a Suite of Aerodynamic Shape Optimization Problems Karla Telidetzki

Master of Applied Science

Graduate Department of Aerospace Engineering

University of Toronto

2014
\end{abstract}

The impact of dimensionality on three aerodynamic optimization cases is studied to determine the effect of the number of geometric design variables. The cases investigated are: (1) drag minimization of an airfoil in transonic inviscid flow, (2) drag minimization through optimizing the twist distribution of a rectangular wing in subsonic inviscid flow, and (3) drag minimization through optimizing the twist distribution and section shapes of a wing in transonic turbulent flow. The optimization algorithm achieves significant drag reductions in all three cases. Valuable insight into the impact of dimensionality is gained, showing that increasing the number of design variables can provide greater flexibility. This flexibility can come at a cost to the convergence of the optimization algorithm, whether that be due to an inability to reduce the optimality significantly, the increased geometric flexibility challenging the mesh movement algorithm, or the unique aerodynamic shapes being unable to achieve converged flow solutions. 


\section{Acknowledgements}

I would like to thank Dr. David W. Zingg for his guidance during the completion of this Master's thesis. His supervision has been invaluable to me, without which I would not have achieved this milestone. I deeply appreciate his support of not only my academic endeavours, but personal and professional achievements as well.

To the students and staff that make up the UTIAS community, thank you for making my experience memorable. UTIAS is not just a place for academic study, and many individuals have contributed to the enrichment of my experience during my time here. The opportunities provided to learn from academic experts and industry leaders are outstanding, and allowed me to learn about many fascinating subjects.

Thank you to my family for their endless support, and for their courage to ask "How is your thesis coming along?". Nigel, thank you for your love and patience. I would not have started, or finished, without your encouragement and support.

The financial support of the Natural Sciences and Engineering Research Council of Canada, the Government of Ontario, the Government of Alberta, and the University of Toronto is gratefully acknowledged. 


\section{Contents}

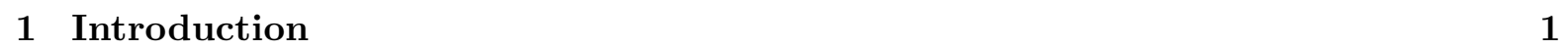

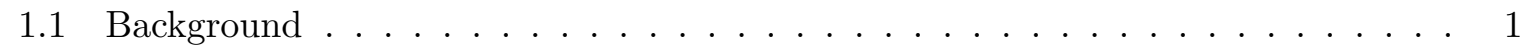

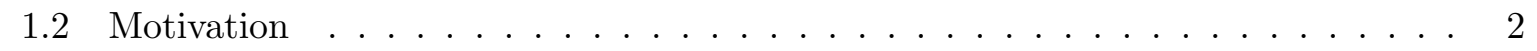

1.3 Literature Review . . . . . . . . . . . . . . . . . . . . . . . 4

$1.3 .1 \quad$ Impact of Dimensionality $\ldots \ldots \ldots \ldots \ldots \ldots$

1.3 .2 Benchmark Problems $\ldots \ldots \ldots \ldots \ldots$

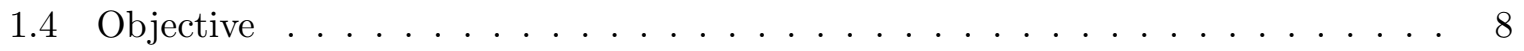

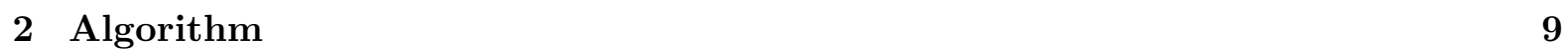

2.1 Geometry Parameterization and Mesh Movement . . . . . . . . . . . . . . . . 9

$2.1 .1 \quad$ B-Spline Curves $\ldots \ldots \ldots \ldots \ldots \ldots$

$2.1 .2 \quad$ B-Spline Curve Fitting . . . . . . . . . . . . . . . . . . 11

2.1 .3 Parameter Correction . . . . . . . . . . . . . . . . . . 12

$2.1 .4 \quad$ B-Spline Surfaces and Volumes . . . . . . . . . . . . . . . . . 13

2.1 .5 Mesh Movement . . . . . . . . . . . . . . . . . . . . . . . . . . 14

2.2 Flow Solver . . . . . . . . . . . . . . . . . . . . . . . . . . . . . 15

2.3 Optimization Algorithm $\ldots \ldots \ldots \ldots \ldots \ldots$

2.3 .1 Gradient Evaluation $\ldots \ldots \ldots \ldots \ldots$. . . . . . . . . . . . . . . . . .

$2.3 .2 \quad$ SNOPT Optimization $\ldots \ldots \ldots \ldots \ldots \ldots$

$\begin{array}{lll}3 & \text { Results } & 22\end{array}$

$3.1 \quad$ Case 1: Symmetrical Airfoil Optimization in Transonic Inviscid Flow . . . . . . . 22

$3.1 .1 \quad$ Optimization Problem $\ldots \ldots \ldots \ldots \ldots \ldots \ldots$. . . . . . . . . . . . . . . . 
3.1 .2 Flow Conditions . . . . . . . . . . . . . . . . 23

3.1 .3 Initial Geometry $\ldots \ldots \ldots \ldots \ldots \ldots \ldots$

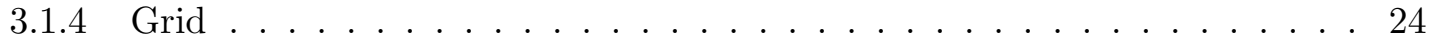

$3.1 .5 \quad$ Optimization Results $\ldots \ldots \ldots \ldots \ldots \ldots$

3.2 Case 2: Twist Optimization of a Rectangular Wing in Subsonic Inviscid Flow . . 31

$3.2 .1 \quad$ Optimization Problem . . . . . . . . . . . . . . . . . . 31

3.2 .2 Flow Conditions $\ldots \ldots \ldots \ldots \ldots \ldots \ldots$

3.2 .3 Initial Geometry $\ldots \ldots \ldots \ldots \ldots \ldots$

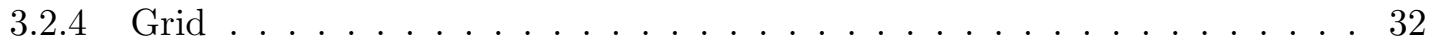

3.2 .5 Optimization Results . . . . . . . . . . . . . . . . . . . 33

3.3 Case 3: Wing Twist and Section Optimization in Transonic Turbulent Flow . . . 35

$3.3 .1 \quad$ Optimization Problem . . . . . . . . . . . . . . . . . . . 35

3.3 .2 Flow Conditions . . . . . . . . . . . . . . . . 36

$3.3 .3 \quad$ Initial Geometry $\ldots \ldots \ldots \ldots$. . . . . . . . . . . . . 36

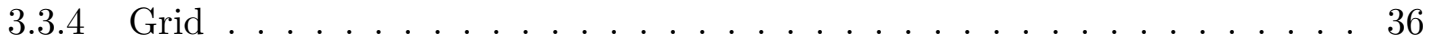

3.3 .5 Optimization Results . . . . . . . . . . . . . . . . . . . . . . . 37

$\begin{array}{|ll|}4 \text { Conclusions and Recommendations } & 41\end{array}$

4.1 Conclusions $\ldots \ldots \ldots \ldots \ldots \ldots \ldots \ldots$

4.2 Recommendations $\ldots \ldots \ldots \ldots \ldots \ldots \ldots \ldots$

\begin{tabular}{ll}
\hline References & 44
\end{tabular} 


\section{List of Tables}

3.1 Grid parameters for NACA 0012 airfoil grid study . . . . . . . . . . . . . . 25

3.2 Results of grid study for NACA 0012 airfoil inflated using 48 streamwise control

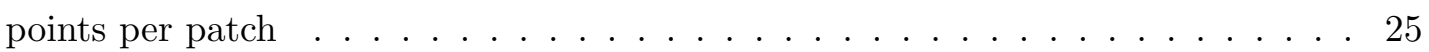

3.3 Grid study for NACA 0012 airfoil optimization on coarse grid . . . . . . . . . . . 30

3.4 Grid study for NACA 0012 airfoil optimization on medium grid . . . . . . . . . . 30

3.5 Grid study for NACA 0012 airfoil optimization on fine grid . . . . . . . . . . . 30

3.6 Coefficients for rectangular wing with NACA 0012 sections on optimization mesh and fine mesh $\ldots \ldots \ldots \ldots \ldots \ldots$. . . . . . . . . . . . . . . . . . . . . . . . . . 


\section{List of Figures}

$2.1 \quad$ Example of colinear control points at airfoil leading edge . . . . . . . . . . . . 12

2.2 Comparison of fitting a NACA 0012 airfoil using B-spline curves with 5 control points, with and without parameter correction $\ldots \ldots \ldots \ldots . \ldots 12$

2.3 Comparison of fitting a NACA 0012 airfoil using B-spline curves with 9 control points, with and without parameter correction $\ldots \ldots \ldots \ldots$. . . . . . 12

2.4 Comparison of fitting a NACA 0012 airfoil using B-spline curves with 14 control points, with and without parameter correction . . . . . . . . . . . . . . 12

2.5 Plot of fit residual between NACA0012 airfoil and B-spline curve before parameter correction . . . . . . . . . . . . . . . . . . . 13

2.6 Effect of pressure switch on optimization of inviscid, transonic NACA 0012 airfoil at zero angle of attack . . . . . . . . . . . . . . . . 18

2.7 Comparison of Mach contours of NACA 0012 airfoil with and without pressure

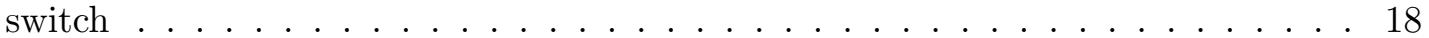

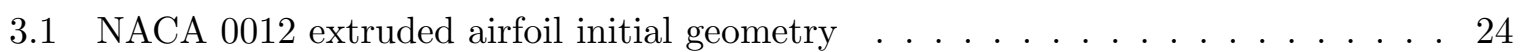

$3.2 \quad$ Coarse grid level for NACA 0012 extruded airfoil $\ldots \ldots \ldots \ldots$. . . . . . . . . . 24

3.3 Comparison of optimization of the NACA 0012 airfoil using Jetstream on fine and medium grids with Vassberg et al. results $[27] \ldots \ldots \ldots \ldots$

3.4 Comparison of Jetstream results on different grid levels for NACA 0012 airfoil. . 25

3.5 Typical convergence histories for NACA 0012 airfoil optimization . . . . . . . . . 27

3.6 Comparison of final optimized airfoil shapes to initial NACA 0012 airfoil . . . . . 27

3.7 Comparison of final coefficient of pressure distributions to initial NACA 0012

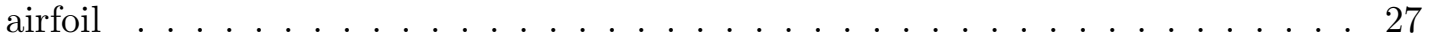


3.8 Comparison of Mach contours for initial NACA 0012 airfoil and final optimized shape with 9 design variables $\ldots \ldots \ldots \ldots \ldots$. . . . . . . . . . . . . .

3.9 Comparison of entropy contours for initial NACA 0012 airfoil and final optimized shape with 9 design variables $\ldots \ldots \ldots \ldots \ldots \ldots$

3.10 NACA 0012 rectangular wing initial geometry . . . . . . . . . . . . . . 32

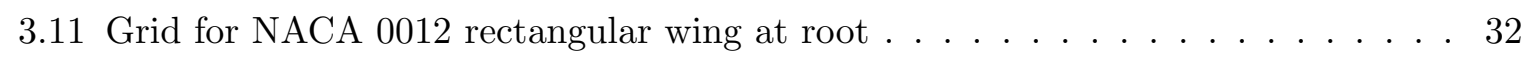

3.12 Effect of change in number of spanwise control points per inboard patch on final drag coefficient for NACA 0012 rectangular wing twist optimization case . . . . . 33

3.13 Effect of change in number of spanwise control points per inboard patch on final span efficiency factor for NACA 0012 rectangular wing twist optimization case . 33

3.14 Effect of change in number of spanwise control points per inboard patch on change in span efficiency factor for NACA 0012 rectangular wing twist optimization case 34

3.15 Typical optimization convergence history for NACA 0012 rectangular wing twist optimization case . . . . . . . . . . . . . . . . . 34

3.16 Lift distribution for NACA 0012 rectangular wing twist optimization case with 11 spanwise control points per inboard patch $\ldots \ldots \ldots$. . . . . . . 34

3.17 Comparison of twist distribution for cases with 5 and 11 spanwise control points per inboard patch to initial twist of NACA 0012 rectangular wing . . . . . . . . . 35

3.18 Initial CRM wing geometry $\ldots \ldots \ldots \ldots \ldots$

3.19 CRM wing drag results - all parameterizations . . . . . . . . . . . . 38

3.20 Optimization convergence histories for CRM wing optimization - 5 streamwise control points per patch . . . . . . . . . . . . . . . . . . 38

3.21 Spanwise lift distribution for CRM wing optimization with 5 streamwise and 7 spanwise control points per patch $\ldots \ldots \ldots \ldots$. . . . . . . . . . . . . .

3.22 Comparison of initial and final surface pressure coefficient distributions for CRM wing optimization with 5 streamwise and 7 spanwise control points per patch . . 39

3.23 Comparison of initial and final airfoil shapes for CRM wing optimization with 5 streamwise and 7 spanwise control points per patch . . . . . . . . . . . 39 


\title{
List of Symbols and Abbreviations
}

\author{
$\alpha \quad$ angle of attack \\ $\boldsymbol{\xi} \quad$ vector of curvilinear coordinates \\ $\Delta c_{j} \quad$ parameter correction variable \\ $\Lambda \quad$ aspect ratio \\ $\mathbf{B}_{i j k} \quad$ vector of grid coordinates for a single B-spline control point \\ $\mathbf{X}$ vector of design variables \\ $\mathcal{N}_{i}^{p} \quad$ B-spline basis function of order $p$ \\ $\xi, \eta, \zeta$ curvilinear coordinates \\ c constraints of optimization problem \\ $C^{p-m-1}$ continuity of knot vector \\ $C_{\mathrm{D}} \quad$ drag coefficient \\ $C_{\mathrm{L}} \quad$ lift coefficient \\ $C_{\mathrm{M}} \quad$ pitching moment coefficient \\ $D_{j} \quad$ surface point of data to be fit \\ e $\quad$ span efficiency factor \\ $f \quad$ objective function of optimization problem
}


$g \quad$ gradient of the objective function of optimization problem

$H \quad$ quasi-Newton approximation to the Hessian of the Lagrangian

$J \quad$ Jacobian of constraints of optimization problem

$L \quad$ total length of the control polygon defined by connecting the control points of the Bspline curve

$L_{s} \quad$ segment length between surface points

$L_{T} \quad$ total length of all surface points

$M \quad$ Mach number

$m \quad$ multiplicity of knot vector

$N \quad$ number of control points in B-spline parameterization

$p \quad$ order of B-spline basis function

$S \quad$ total number of airfoil surface points

$t_{i} \quad$ knot vector component

$w_{j} \quad$ component of chord-length parameterization

$x, y, z$ Cartesian coordinates

$X_{j} \quad$ component of B-spline fitted curve

$Y_{j} \quad$ normalized tangent vector to B-spline curve

CAD Computer Aided Design

CFD Computational Fluid Dynamics

CRM Common Research Model

FFD Free-Form Deformation

FGMRES Flexible Generalized Minimal Residual 
GMRES Generalized Minimal Residual

IATA International Air Transport Association

MAC Mean Aerodynamic Chord

NURBS Non-Uniform Rational B-Spline

RANS Reynolds-Averaged Navier-Stokes

SATs Simultaneous Approximation Terms

SBP Summation-by-Parts

SLSQP Sequential Least Squares Quadratic Programming

SNOPT Sparse Nonlinear Optimizer 



\section{Chapter 1}

\section{Introduction}

\section{$1.1 \quad$ Background}

Growth in air traffic is driving the need for more fuel efficient aircraft to reduce greenhouse gas emissions and fuel costs for airlines. In 2009, the International Air Transport Association (IATA) outlined a set of targets for the reduction of carbon dioxide emissions produced by commercial aircraft. The goals set forth by IATA require: an improvement in fuel efficiency of $1.5 \%$ per year from 2009-2020; carbon-neutral growth from 2020; and a $50 \%$ reduction in emissions by 2050, compared to 2005 [14. A four-pillar strategy has been identified as the means to achieve these ambitious targets:

1. Improved technology

2. Effective operations

3. Efficient infrastructure

\section{Positive economic measures}

The last three pillars consist of operations changes, infrastructure updates and government policy. The first pillar, improved technology, is expected to provide the greatest reduction in fuel burn and emissions. This could include unconventional aircraft, new engine types and the use of biofuels. 
Aerodynamic shape optimization has emerged to address these issues, motivated by the need to reduce greenhouse gas emissions and increase fuel efficiency. Combining Computational Fluid Dynamics (CFD) with numerical optimization methods allows designers to effectively explore the design space. The use of a computer algorithm allows the designer to focus on defining the priorities, objectives and constraints for the problem, rather than the aerodynamic shape itself. Aerodynamic shape optimization has four key components:

1. A geometry parametrization which defines the design variables that control the change in geometry

2. A mesh movement algorithm that moves the computational grid with the change in geometry

3. A flow solver

4. An optimization algorithm

Each component has a key role to play in the final solution and how efficiently that final solution is obtained. There are several different methods that can be used for each component. The geometry parametrization, for example, can be accomplished using a CAD package, basis splines, surface mesh nodes, or free-form deformation. When all four component are combined, an aerodynamic shape optimization algorithm is created. This algorithm can be used to optimize airfoils, wings, and more complex blended wing body configurations. It is useful for optimizing conventional geometries, as well as discovering new unconventional configurations.

\subsection{Motivation}

The Drag Prediction Workshops were created by the CFD community to allow an assessment of the performance of different CFD algorithms for practical aerodynamic flows. The aerodynamic shape optimization community is undertaking a similar endeavour. In doing so, a suite of increasingly complex benchmark problems is being developed. At present there are four cases constituting the suite of benchmark problems, which are used to compare different algorithms. 
The first case is drag minimization of a two-dimensional NACA 0012 airfoil in transonic inviscid flow. The second case is drag minimization of a two-dimensional RAE 2822 airfoil in transonic turbulent flow, subject to lift and pitching moment constraints. The third case is drag minimization through optimizing the twist distribution of a three-dimensional rectangular wing compromised of NACA 0012 sections in subsonic inviscid flow, subject to a lift constraint. The final case is drag minimization through optimizing the sections and twist distribution of the blunt-trailing-edge Common Research Model (CRM) wing in transonic turbulent flow, subject to lift and pitching moment constraints.

This thesis explores three of the four benchmark cases (all but the two-dimensional RAE 2822 case) using Jetstream, a high-fidelity aerodynamic shape optimization algorithm for threedimensional turbulent flows [20, 21, 11]. For each case, a systematic study on the impact of the number of design variables on optimizer performance is completed. With the method of geometry parameterization used for this study, the number of design variables increases directly with the number of points controlling the geometry. As the number of geometric control points increases, the problem has greater geometric flexibility. However, this comes at a cost to the efficiency of the optimization algorithm. The optimization algorithm used with Jetstream is called SNOPT (Sparse Nonlinear OPTimizer), and was developed by Gill, Murray and Saunders 8. It is a gradient-based optimizer capable of finding a local optimum for a constrained optimization problem. The performance of SNOPT is greatly impacted by the choice of design variables as well as the constraints placed on them. It will perform most efficiently for problems that have a lower number of design variables and/or relatively few degrees of freedom. Since the performance of SNOPT can degrade as the number of design variables increases, this suggests that there is a preferred number of design variables that will allow a significant decrease in the objective function while still maintaining good performance. The results of this study provide guidance on how to best parametrize the geometry and set up the optimization problem for the benchmark cases. The knowledge gained can be applied to more general optimization problems. 


\subsection{Literature Review}

\subsubsection{Impact of Dimensionality}

Andreoli et al. [2] developed a free-form deformation technique with Bezier volumes and applied it to the optimization of a three-dimensional wing in transonic flow. They used two gradientfree optimization algorithms: the Nelder-Mead simplex method and a genetic algorithm. For each optimization method, they looked at the impact of gradually increasing the number of design variables via degree elevation during the optimization. This is accomplished by allowing the optimizer to solve for a given number of iterations, then applying degree elevation to the geometry at that point and continuing the optimization with the higher number of control points. They compared this result with that obtained using the finest parametrization only. They increased the number of control points in the streamwise direction from 6 to 9 , increasing by one control point each time, and compared to the results using 9 streamwise control points initially. For the simplex methods, the degree elevation process produces an improvement in the objective function (the drag coefficient) and an improvement in convergence speed. When using a genetic algorithm, the effect of the degree elevation is less clear. Gradually increasing the number of control points produces faster convergence, but starting with the finest parametrization produces a better objective function.

Buckley and Zingg [4] looked at the impact of the number of design variables for multipoint optimization of an airfoil in subsonic flow conditions. The gradient-based optimizer SNOPT was used. They increased the number of design variables from 12 to 30, and saw only a slight trend of better on-design performance as the number of design variables increased. The difference between the coarsest and finest parametrization results was only $0.6 \%$. As the number of design variables increased, the computational expense also increased.

Zingg et al. 29] investigated the effect of the number of design variables on the convergence of a gradient-based and a genetic algorithm. They parameterized a two-dimensional airfoil with 15, 25, and 45 control points. This corresponds to 9, 19, and 35 design variables, which includes angle of attack. As the number of design variables increases, the number of function

evaluations required by the optimizer also increases. For the gradient-based algorithm, the number of function evaluations roughly doubles as the number of design variables doubles. The 
genetic algorithm sees a much higher increase in the number of function evaluations as the number of design variables increases, but the ratio is not consistent.

Han and Zingg [9] applied an evolutionary geometry parametrization using B-splines and refined the parametrization through a sequence of optimizations. The idea is similar to the degree elevation process performed by Andreoli et al., but the use of B-splines does not require degree elevation to increase the number of design variables. The optimization algorithm used is again SNOPT. This technique produced an improvement in the objective function, but at significant computational expense.

Kumar et al. [16], using fourth-order NURBS parameterization, investigated the results of aerodynamic shape optimization with a changing number of design variables. They used a gradient-based optimizer to obtain maximum lift at a fixed angle of attack with Reynolds numbers of $10^{3}$ and $10^{4}$. At the lower Reynolds number $\left(10^{3}\right)$, performing optimization with 13 , 39, and 61 control points showed that the highest lift coefficient was obtained using 13 control points, a lift coefficient of 0.847 , compared with 0.275 and 0.284 for 39 and 61 control points, respectively. They suggest that this is due to multimodality, and that a richer design space creates more local minima. When they instead progressively increased the design space, using the optimal shape from the previous parameterization as an initial guess, the lift coefficient increased each time, from 0.847 with 13 control points to 1.356 with 61 control points. At the higher Reynolds number $\left(10^{4}\right)$, they performed optimization with 13, 27, and 39 control points and used the optimal shape from the previous parameterization as the initial guess for the higher parameterization. The highest lift coefficient was obtained with 39 control points.

Thokola and Martins [25] applied variable-complexity methods to aerodynamic shape optimization of a two-dimensional airfoil. They employed variable-fidelity and variable-parameterization. Of interest to this thesis is the variable-parameterization results. Their method involved comparing the results of optimizing solely using the highest parameterization to the results of optimizing a problem with a reduced parameterization, and then using that optimized result as the initial condition for the highest parameterization. They showed that both methods converged to the same optimum, with the variable parameterization approach taking more computational time in one case, and less computational time in another. The design space used was small, with the highest parameterization consisting of 15 design variables. 


\subsubsection{Benchmark Problems}

The two-dimensional NACA 0012 benchmark case is based on work done by Vassberg et al. [27. This work presented the results of a systematic study on the impact of dimensionality. Using a Bezier curve parameterization, the number of design variables was increased from 0 to 36 , and the impact on the final optimized results was analyzed. As the number of design variables increased, the final drag coefficient decreased from 468.9 drag counts for the initial geometry to 103.8 drag counts for the final optimized geometry with 36 design variables.

Bisson et al. [3] presented the results of the two-dimensional NACA 0012 and three-dimensional rectangular wing twist cases. The geometry parametrization is accomplished using B-splines and the mesh movement using radial basis functions. The flow solver is a cell-centered finite volume Reynolds-averaged Navier-Stokes (RANS) solver and the optimization algorithm used is SNOPT. The parametrization and optimization algorithm are similar to those used in Jetstream. For the two-dimensional NACA 0012 case, four different geometry parametrizations were studied: 16, 24, 32, and 48 control points. As the number of control points increased, a lower optimal drag was obtained. Their results also show the importance of performing a grid convergence study on both the initial and final geometries. While the initial grid study showed little difference in drag calculated on the medium and fine grid levels, a final grid study showed a significant drop in the drag coefficient on finer grid levels. For the three-dimensional rectangular wing twist case, only a single geometry parameterization was studied. They were able to produce a near-elliptical lift distribution with the optimized shape. A grid study showed that the same span efficiency factor was obtained when comparing optimization on a coarse grid to optimization on a fine grid, with both shapes analyzed by performing a flow solve on the fine grid.

Carrier et al. [5] analyzed the two-dimensional NACA 0012 and three-dimensional CRM cases. They used several local, gradient-based optimization algorithms, including a Sequential Least Squares Quadratic Programming (SLSQP) algorithm that is similar to SNOPT. For the two-dimensional NACA 0012 case, three different types of geometry parameterization were used: Bezier curves; B-spline curves; and a full parameterization where the $z$-coordinate of each surface mesh node is used as a design variable. An initial analysis comparing the Bezier and B- 
spline parameterizations using 6 control points showed the B-spline parameterization obtained a significantly lower optimized drag coefficient of 150 drag counts, compared to 350 drag counts for the Bezier parameterization. When the control points for the Bezier parameterization are redistributed based on an a posteriori analysis of the results, a better solution of 200 drag counts is obtained. This shows the location of the control points has a significant impact on the results, as well as the parameterization method itself. A second analysis used a hierarchy of parameterizations based on Bezier curves, ranging from 6 to 96 design variables, with the parameterizations consistent with those from Vassberg et al. [27]. This was first performed using a conjugate gradient optimization algorithm. As the number of design variables is increased from 6 to 36, better optimal results are obtained, from 360 to 77.6 drag counts. However, past 36 design variables, a better optimum is not achieved. As the number of design variables increases, the problem becomes stiffer and the optimizer is not able to improve performance. The three highest dimension parameterizations (36, 64 and 96 design variables) were analyzed using the SLSQP algorithm. With this optimization algorithm, increasing the number of design variables does produce a better optimal result. A third analysis looked at the full parameterization, which did not produce optimal results as good as those achieved using the Bezier parameterization in the second analysis. For the three-dimensional CRM case, 6 optimizations were performed with various chordwise and/or spanwise refinements. As the number of chordwise control points is increased, the wave drag is decreased. The number of spanwise control sections impacts the induced drag component, with a fine parameterization required to minimize the induced drag. These optimizations were performed allowing twist and camber modifications, such that the internal wing volume remains constant. This differs from the formal problem description, which only requires the internal volume be equal to or greater than the initial volume, allowing section changes.

Amoignon et al. [1] analyzed the two-dimensional NACA 0012 case. The geometry was parameterized using Free-Form Deformation (FFD). As the number of design variables is increased, the final drag coefficient is reduced from 475 drag counts for the initial shape to 113.8 drag counts for the final shape with 41 design variables. However, many of these cases produced lift coefficients that were non-zero. 


\subsection{Objective}

The objective of this thesis is to analyze a suite of standard aerodynamic optimization test cases, looking at the impact of the number of design variables. The results will contribute to the development of the aerodynamic shape optimization benchmark cases, as well as provide insight into the impact of dimensionality. Three of the four benchmark cases will be analyzed:

1. Drag minimization of a two-dimensional NACA 0012 airfoil in transonic inviscid flow

2. Drag minimization through optimizing the twist distribution of a three-dimensional rectangular wing with NACA 0012 sections in subsonic inviscid flow, subject to a lift constraint

3. Drag minimization through optimizing the sections and twist distribution of the blunttrailing-edge Common Research Model (CRM) wing in transonic turbulent flow, subject to lift and pitching moment constraints 


\section{Chapter 2}

\section{Algorithm}

\subsection{Geometry Parameterization and Mesh Movement}

Geometric parameterization is employed to have a balance between the number of design variables and the ability to represent a wide variety of shapes. Numerical optimization algorithms can only handle a finite set of variables, which requires that a geometry parameterization be used to reduce the aerodynamic shape to a finite number of design variables. Several different geometry parameterization techniques have been used for aerodynamic shape optimization, each having its advantages and disadvantages. A good parameterization should be able to approximate a wide variety of aerodynamic shapes while using as few design variables as possible. The parameterization in Jetstream uses B-spline tensor volumes to approximate not only the surface, but the entire multi-block structured computational mesh, and was developed by Hicken and Zingg [11]. As a result, the geometry parameterization is integrated with the mesh movement scheme. This section will introduce how B-spline curves and volumes are utilized to fit the surfaces and mesh blocks.

\subsubsection{B-Spline Curves}

A B-spline curve of order $p$ is composed of a linear combination of control points and basis functions:

$$
\mathbf{X}(\xi)=\sum_{i=1}^{N} \mathbf{B}_{i} \mathcal{N}_{i}^{(p)}(\xi)
$$


where $\mathbf{B}_{i}$ are the coordinates of the de Boor control points, and $\mathcal{N}_{i}^{(p)}(\xi)$ are the B-spline basis functions of order $p$ (degree $p-1)$. There are $N$ control points used for a given parameterization. The number of control points used will affect the accuracy with which the B-spline curve can fit a given curve, with the minimum number of control points equal to $p$. A more complex shape will require a higher number of control points to achieve a certain B-spline fitting accuracy. The basis functions are defined according to the Cox-de-Boor recursion formula [7]:

$$
\begin{gathered}
\mathcal{N}_{i}^{(1)}(\xi)= \begin{cases}1 & \text { if } t_{i} \leq \xi \leq t_{i+1} \\
0 & \text { otherwise }\end{cases} \\
\mathcal{N}_{i}^{(p)}(\xi)=\frac{\xi-t_{i}}{t_{i+p-1}-t_{i}} \mathcal{N}_{i}^{(p-1)}(\xi)+\frac{t_{i+p}-\xi}{t_{i+p}-t_{i+1}} \mathcal{N}_{i+1}^{(p-1)}(\xi)
\end{gathered}
$$

and the basis functions are defined along a knot vector

$$
\mathbf{T}=\left(t_{1}, t_{2}, \ldots, t_{p-1}, t_{p}, t_{p+1}, \ldots, t_{N-1}, t_{N}, t_{N+1}, \ldots, t_{N+p}\right)
$$

where the knot vector has $N+p$ elements, equal to the number of control points $(N)$ plus the order of the basis functions $(p)$. The multiplicity of the knots affects the continuity of the curve, such that a knot with multiplicity $m$ has a continuity of $C^{p-m-1}$. If the multiplicity is equal to the order of the B-spline curve $(m=p)$, the curve has $C^{-1}$ continuity, or a discontinuity, at that point. Enforcing this type of multiplicity at the endpoints of the knot vector ensures the control points coincide with the data points at the beginning and end of the curve.

The order of the basis functions and the definition of the knot vector both affect the B-spline curve. In this work, all B-spline basis functions are fourth order $(p=4)$. This means that the minimum number of control points that can be used is $N=4$, and a multiplicity of $m=4$ is enforced at the end points of the knot vector. The knot vector can be defined in different ways. A uniform knot vector would have evenly spaced knot values, such that $t_{i}-t_{i-1}$ is constant for all values of $i$. With this type of knot vector, enforcing multiplicity of knots is not possible. Since we wish to have the endpoints of the curve pass through certain control points, it is necessary to have a nonuniform knot vector. 


\subsubsection{B-Spline Curve Fitting}

The B-Spline curves presented in the previous section must be expanded in order to be useful for fitting practical aerodynamic curves and surfaces. In order to fit a surface using a Bspline curve, the surface must be parameterized and the knot vector must be set. A discrete parameterization is required, so in place of $\xi$ in the equations above, $w_{j}$ is used. A chord length parameterization is used

$$
w_{1}=0, w_{j}=\frac{\sum_{s=1}^{j-1} \sqrt{L_{s}}}{L_{T}} \text { where } j=2, \ldots, S-1, w_{S}=1
$$

where $S$ is the total number of airfoil surface points, $L_{s}$ is the segment length between surface points, and $L_{T}$ is the total length of all surface points.

A nonuniform knot sequence is used, given by [9]:

$$
t_{i}= \begin{cases}0 & \text { if } 1 \leq i \leq p \\ \frac{1-\cos \left(\frac{i-p}{N-p+1} \pi\right)}{2} & \text { if } p+1 \leq i \leq N \\ 1 & \text { if } N+1 \leq i \leq N+p\end{cases}
$$

Once the knot vector and parameterization are set, the B-spline basis functions can be calculated. In order to determine the locations of the control points, the following must be minimized

$$
\min \sum_{j=1}^{S}\left\|D_{j}-X_{j}\right\|
$$

where $D_{j}$ are the surface points of the data to be fit, and $X_{j}$ is the fitted curve, as defined by equation 2.1. In order to ensure the leading edge of the airfoil is smooth, the control points closest to the leading edge should be colinear, as shown in Figure 2.1. This can be accomplished by moving a control point after the minimization, however this will change the fitted shape that was obtained. Another way to accomplish this is to do a constrained minimization that sets the colinearity through the least squares solution. In the algorithm, the colinearity is set outside the minimization, and then the fit is adjusted using parameter correction as described in the next section. 


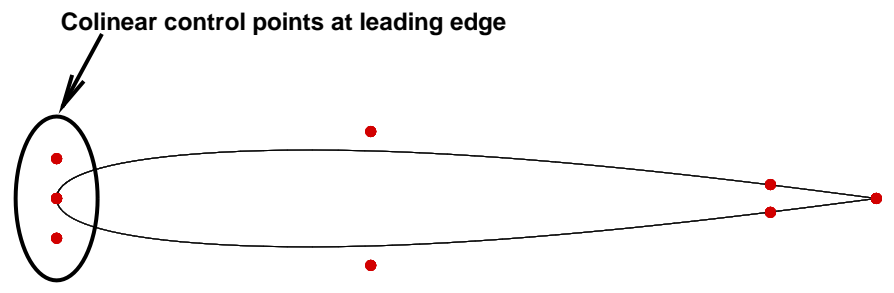

Figure 2.1: Example of colinear control points at airfoil leading edge

\subsubsection{Parameter Correction}

With the parameterization method described in the previous sections, the vectors $D_{j}-X_{j}$ are not perpendicular to the tangent of the fitted curve $X_{j}^{\prime}$. A better fit can be obtained by changing the values of the parameters $w_{j}$ through an iterative parameter correction procedure, such as that suggested by Hoschek [13]

$$
\begin{gathered}
\bar{w}_{j}=w_{j}+\Delta c_{j} \frac{t_{N}-t_{1}}{L} \\
\Delta c_{j}=\left(D_{j}-X_{j}\right) Y_{j}
\end{gathered}
$$

where $L$ is the total length of the control polygon defined by connecting the control points, and $Y_{j}$ is the normalized tangent vector, found by calculating the derivative of the B-spline curve. In order to demonstrate the effect of the parameter correction, it is applied to the fit of one half of the symmetrical NACA0012 airfoil and iterated until the residual drops below $10^{-5}$, the maximum number of iterations (1000) is reached, or the residual stops changing.

Figures 2.2 to 2.4 show how the impact of the parameter correction on the quality of the fit depends on the number of control points. In practice, the parameter correction is implemented on curves that compose a three-dimensional extruded airfoil or wing. This is done until the residual drops below a tolerance of $1 \times 10^{-6}$ or 1000 iterations are performed. An extruded 

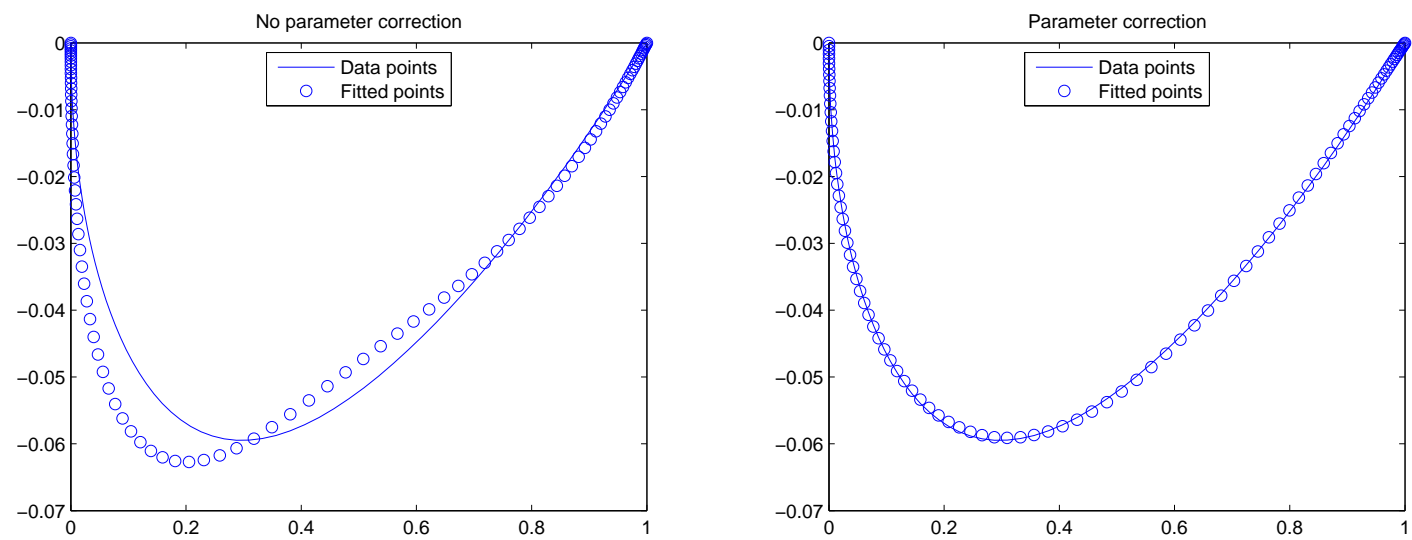

Figure 2.2: Comparison of fitting a NACA 0012 airfoil using B-spline curves with 5 control points, with and without parameter correction
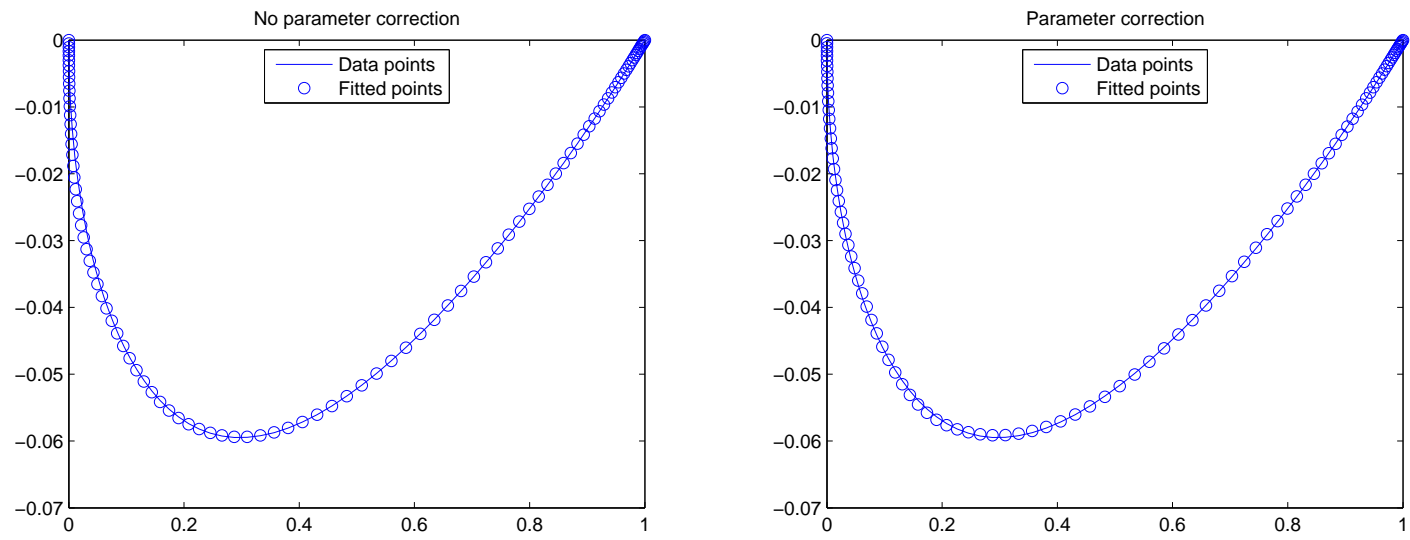

Figure 2.3: Comparison of fitting a NACA 0012 airfoil using B-spline curves with 9 control points, with and without parameter correction
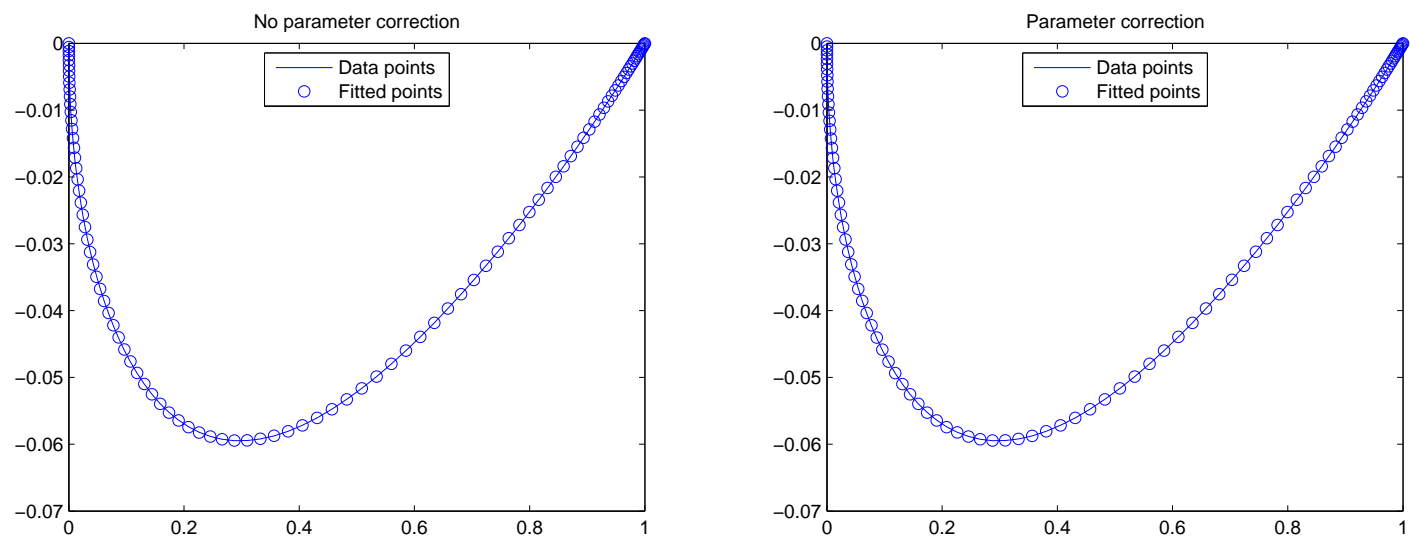

Figure 2.4: Comparison of fitting a NACA 0012 airfoil using B-spline curves with 14 control points, with and without parameter correction 


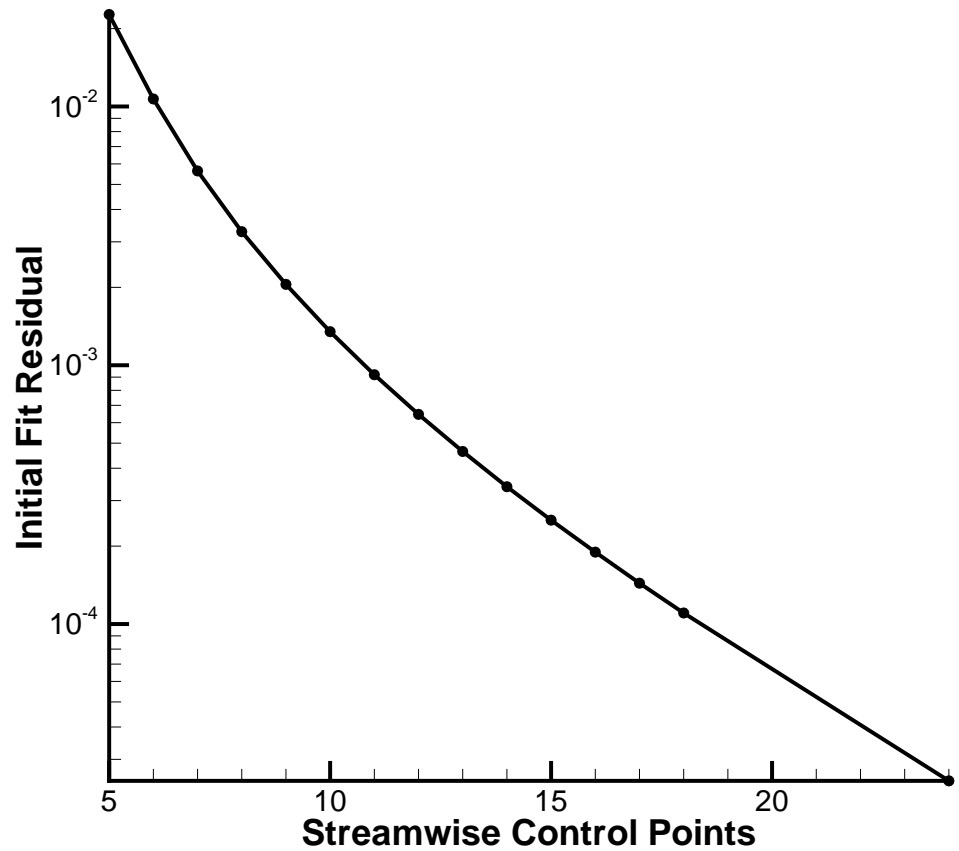

Figure 2.5: Plot of fit residual between NACA0012 airfoil and B-spline curve before parameter correction

airfoil is composed of two curves, one which defines the upper surface and one which defines the lower surface, that are extruded into space. For a NACA 0012 airfoil, these curves are symmetrical about the chord. When 5 streamwise control points are used to parameterize each surface (upper and lower) of the extruded NACA 0012 airfoil, the initial residual is $2.3 \times 10^{-2}$, and after 1000 iterations of the parameter correction it is dropped to $8.4 \times 10^{-5}$. When 9 streamwise control points are used, the residual begins at $2.1 \times 10^{-3}$ and drops to $4.5 \times 10^{-5}$ after 1000 iterations. Figure 2.5 shows how the initial residual changes with the number of streamwise control points. As the number of control points is increased, the initial fit residual decreases.

The parameter correction is limited in how much it can efficiently decrease the residual of the curve fit. For example, when using 5 streamwise control points, the residual will not drop much below $1 \times 10^{-4}$, even if allowed to continue for 100,000 iterations. When 14 streamwise control points are used, after 100,000 iterations the residual will drop from $3.39 \times 10^{-4}$ to $5 \times 10^{-6}$. The parameter correction can only drop the residual approximately two orders of 
magnitude. Using 100,000 iterations does not take much time in this case, because only a single curve is being fit, but if multiple curves or the entire grid had the parameter correction applied, it would not be possible to allow it to iterate so long.

Two of the cases investigated in this thesis use the parameter correction for B-spline curve fitting to improve the initial geometry, as their geometries are composed of an extruded airfoil in the two-dimensional case and an extruded airfoil with a pinched tip in the three-dimensional case. The parameter correction is not applied to the more complicated Common Research Model wing, as it is more costly to apply in three-dimensions.

\subsubsection{B-Spline Surfaces and Volumes}

The concepts that were explained in the previous sections for B-spline curves can be extended to produce B-spline surfaces and B-spline volumes. This allows parameterization of the aerodynamic surface and the entire computational grid. For a B-spline volume, the curve definition in equation 2.1 becomes

$$
\mathbf{X}(\boldsymbol{\xi})=\sum_{i=1}^{N_{i}} \sum_{j=1}^{N_{j}} \sum_{k=1}^{N_{k}} \mathbf{B}_{i j k} \mathcal{N}_{i}(\xi) \mathcal{N}_{j}(\eta) \mathcal{N}_{k}(\zeta)
$$

where $\mathbf{X}(\boldsymbol{\xi})$ represents the coordinates of the nodes of the computational mesh as a function of curvilinear coordinates $\boldsymbol{\xi}=(\xi, \eta, \zeta)$. The basis functions calculated in the $\xi$ direction, while holding $\eta$ and $\zeta$ constant, are

$$
\begin{gathered}
\mathcal{N}_{i}^{(1)}(\xi ; \eta, \zeta)= \begin{cases}1 & \text { if } t_{i}(\eta, \zeta) \leq \xi \leq t_{i+1}(\eta, \zeta) \\
0 & \text { otherwise }\end{cases} \\
\mathcal{N}_{i}^{(p)}(\xi ; \eta, \zeta)=\frac{\xi-t_{i}(\eta, \zeta)}{t_{i+p-1}(\eta, \zeta)-t_{i}(\eta, \zeta)} \mathcal{N}_{i}^{(p-1)}(\xi ; \eta, \zeta)+\frac{t_{i+p}(\eta, \zeta)-\xi}{t_{i+p}(\eta, \zeta)-t_{i+1}(\eta, \zeta)} \mathcal{N}_{i+1}^{(p-1)}(\xi ; \eta, \zeta)
\end{gathered}
$$


with similar expressions for the $\eta$ and $\zeta$ directions. The knot vector, $t_{i}(\eta, \zeta)$, is a spatially varying function that depends on $\eta$ and $\zeta$. The basis functions are defined along a knot vector

$$
t_{i}(\eta, \zeta)=(1-\eta)(1-\zeta) t_{i}(0,0)+\eta(1-\zeta) t_{i}(1,0)+(1-\eta) \zeta t_{i}(0,1)+\eta \zeta t_{i}(1,1)
$$

where $t_{i}(0,0), t_{i}(1,0), t_{i}(0,1)$ and $t_{i}(1,1)$ are the edge knot values, which are constant. As with the B-spline curve, the surface must be parameterized, and a chord-length parameterization is used. The system is solved using a least-squares fitting, with the block edges fit first, followed by the surfaces, and finally the internal volume control points. The final B-spline volume mesh maintains the relative spacing of the original computational mesh, but is several orders of magnitude smaller. Without this property, areas with high curvature would not be accurately resolved.

\subsubsection{Mesh Movement}

In order for aerodynamic shape optimization to occur, geometry changes must be accommodated by both the parameterization and the mesh movement schemes. By fitting the computational mesh with B-spline volumes, changes in the control points on the B-spline surface can be propagated through the mesh using a method based on the principles of linear elasticity, which was adapted from the work of Truong et al. [26] by Hicken and Zingg [12]. Using a method based on linear elasticity is typically computationally expensive; however, the control mesh made up of the B-spline volume control points is up to two orders of magnitude smaller than the full computational mesh, allowing the computational time to be much lower.

The volumes of the control mesh are treated as linear elastic solids that are isotropic and homogeneous, with a Poisson's ratio of $\nu=-0.2$ to prevent a high aspect ratio. The Young's modulus is proportional to the inverse of the cell volume. To accommodate large shape changes and improve robustness, the mesh movement occurs in increments. This work uses $m=5$ increments, but if mesh movement problems occur, it is possible to use more increments. The intermediate control points are related to their initial $b_{s}^{(0)}$ and final $b_{s}^{(m)}$ values using a linear 
relationship

$$
b_{s}^{(i)}=\frac{i}{m}\left(b_{s}^{(m)}-b_{s}^{(0)}\right)+b_{s}^{(0)}, \quad i=1, \ldots, m .
$$

Equation 2.14 is discretized on the control mesh using a finite-element method. The resulting linear system is solved using the conjugate gradient method preconditioned with ILU(1) [18], with the convergence criterion being a reduction in the $L_{2}$ norm to a relative tolerance of $10^{-12}$. The fine mesh is then updated based on the control mesh using an algebraic approach that is based on the B-spline volume basis functions.

\subsection{Flow Solver}

The flow solver is one of the key components of any aerodynamic shape optimization algorithm. The flow solver should be efficient, accurate and robust. Accuracy is required to allow the optimizer to use the information from the flow solver to produce an optimum solution. Efficiency is required as the optimizer will perform many flow solves through the course of an optimization. Robustness is necessary to accommodate significant geometry changes. This section presents an overview of the parallel three-dimensional multi-block structured solver used in Jetstream.

The flow solver uses the Newton-Krylov method to obtain high-fidelity flow solutions for use within the aerodynamic shape optimization algorithm. The flow solver was developed by Hicken and Zingg [10] for the three-dimensional Euler equations, and by Osusky and Zingg 22 for the three-dimensional RANS equations. The flow solver algorithm solves the three-dimensional RANS equations for viscous turbulent flows, and the Euler equations for inviscid flows. The RANS equations are fully coupled with the Spalart-Allmaras one-equation turbulence model. The governing equations are discretized using second-order Summation-by-Parts (SBP) operators. Boundary conditions and block interfaces use Simultaneous Approximation Terms (SATs). An implicit Euler time marching scheme is applied with an increasing time step, eventually becoming an inexact-Newton method, producing a large, sparse system of linear equations that is solved using flexible GMRES (FGMRES) . An approximate Newton start-up phase is used to provide the initial iterate for the inexact-Newton phase. 


\subsection{Optimization Algorithm}

The optimization algorithm used is called SNOPT (Sparse Nonlinear OPTimizer) and was developed by Gill, Murray and Saunders [8]. It is a gradient based optimizer, capable of finding a local optimum for a constrained optimization problem of the form

$$
\begin{array}{r}
\operatorname{minimize} f(x), x \in \mathbb{R}^{n} \\
\text { subject to } c_{i}(x)=0, i \in \mathrm{E} \\
c_{i}(x) \geq 0, i \in \mathrm{I}
\end{array}
$$

where the vector, $x$, contains the design variables, typically coordinates of the geometric control points and angle of attack. The objective function, $f(x)$, is often the drag coefficient or the ratio of drag to lift. The vector, $c(x)$, contains the constraint functions, which can be linear or nonlinear and are expressed using an equality $(i \in \mathrm{E})$ or inequality $(i \in \mathrm{I})$.

On large problems, SNOPT performs most efficiently if only some of the variables enter the problem nonlinearly or if there are relatively few degrees of freedom at a solution (meaning many of the inequality constraints are at their bounds). For an aerodynamic shape optimization problem, where the lift and drag coefficients depend on the geometry, all the design variables will enter the problem nonlinearly, as lift and drag are nonlinear functions of the design variables. It is therefore reasonable to expect that as the number of design variables increases, the convergence of the optimizer will be affected, as demonstrated by Zingg et al. [29]. In addition, the presence of more design variables can impact the convergence of the mesh movement algorithm or produce geometries that cannot achieve a converged flow solution. When either of these situations occur, SNOPT must find a way back into a region where these functions are defined by shortening the step length it takes along the search direction. If it is not able to get back into a region with defined functions (meaning that both the mesh movement algorithm and the flow solver converge) using this method, the algorithm will terminate. 


\subsubsection{Gradient Evaluation}

To use a gradient-based optimization method, the gradient must be computed accurately and efficiently. Using finite differencing is not possible for the problems in question, as there are too many design variables, and it would be prohibitively inefficient. Pironneau [24] and Jameson [15] proposed the adjoint method, which allows the gradient to be computed at a cost that is virtually independent of the number of design variables. The present work uses the discrete adjoint method, rather than the continuous form. The mesh movement and flow residual equations are treated as nonlinear constraints that are solved outside of SNOPT. These nonlinear constraints are linearized analytically to form the adjoint gradient.

The flow Jacobian matrix is formed by linearizing the components of the discrete flow residual, including the viscous and inviscid fluxes, the numerical dissipation, the turbulence model, and the boundary conditions. This linearization was completed by Hicken and Zingg [11] and Osusky [19]. The pressure switch was not linearized in the algorithm developed by Hicken and Zingg, but was instead treated as a constant in the evaluation of the flow Jacobian. Osusky introduced the full linearization. The pressure switch is used for capturing shocks, and is important to use for transonic flows. It was shown that omitting the pressure switch from the gradient only introduces small errors, which is not problematic for problems that are shock-free at the final geometry. However, one of the cases investigated in this thesis is inviscid and transonic, and the geometries produced are not shock-free. For this case, it was found that if the pressure switch is turned on, but its linearization is not included, the total gradient is inaccurate and the optimization algorithm cannot converge. If the pressure switch is turned off, the drag and lift coefficients obtained are inaccurate. Figure 2.6 shows the effect of the pressure switch on the drag minimization of a two-dimensional NACA 0012 airfoil in inviscid, transonic flow at zero angle of attack with a lift constraint of zero. This is performed on the algorithm Optima2D, which does not include the linearization of the pressure switch. In order to achieve a converged optimization, the pressure switch was turned off to prevent having an inaccurate gradient. When the final optimized geometries were analyzed by performing a flow solve with the pressure switch turned on, the drag coefficients obtained were shown to be much higher than those obtained without the pressure switch, and the lift constraint was no longer 


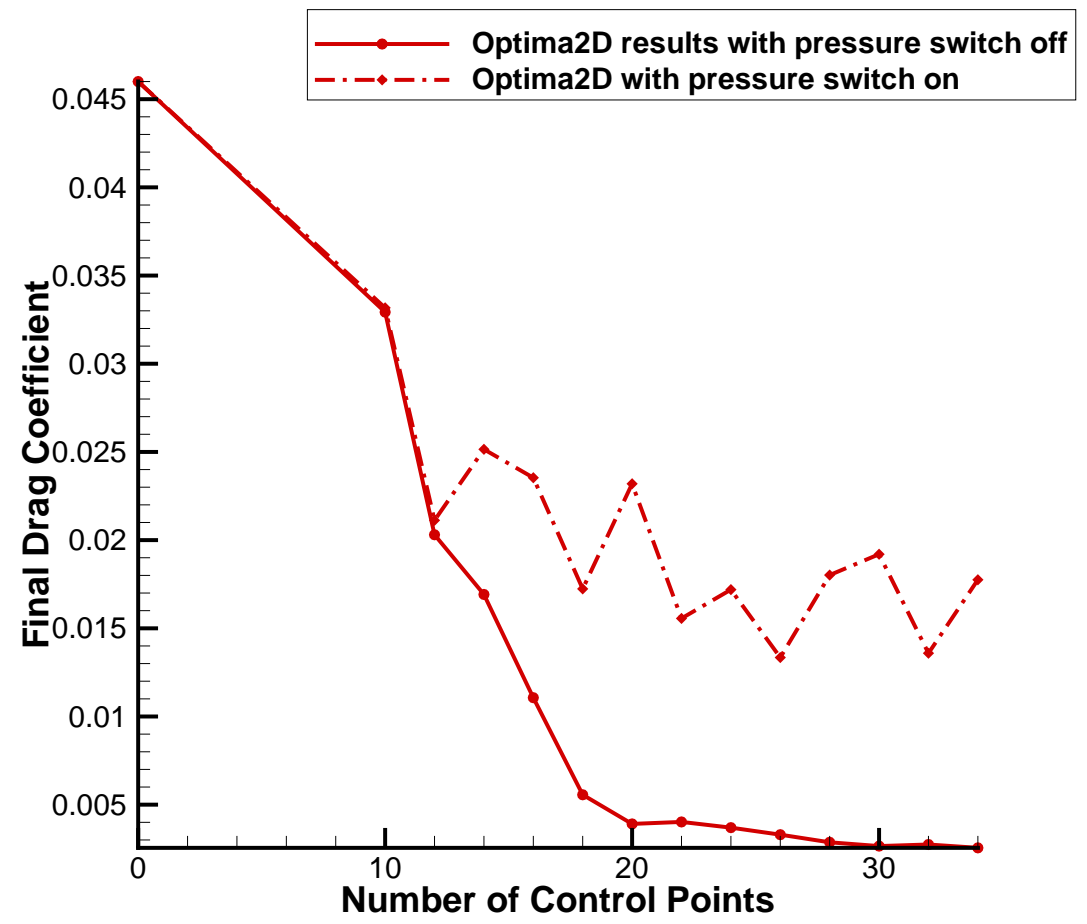

Figure 2.6: Effect of pressure switch on optimization of inviscid, transonic NACA 0012 airfoil at zero angle of attack

met.

Figure 2.7 shows a Mach contour for the final optimized shape of the airfoil using 20 design variables. Without the pressure switch on, this contour looks symmetrical, and the lift constraint is met. When this same geometry is analyzed with the pressure switch on, it is clear the flow is no longer symmetrical across the airfoil chord, and the lift constraint is not met. There is a shock on the upper surface of the airfoil. Although the airfoil is symmetric, it is producing a lifting, or non-unique, solution. Ou et al. [23] analyzed four different symmetrical airfoils, showing that they exhibited non-unique solutions in a range of transonic Mach numbers. The Mach number, $M=0.85$, used for this pressure switch analysis falls within this range. Further analysis of the non-unique solutions produced by this case is recommended.

In summary, the pressure switch is necessary to accurately capture the flow conditions at transonic Mach numbers. If the pressure switch is not linearized and included in the flow Jacobian, the resulting gradient may be so inaccurate that it prevents the optimization algorithm from achieving a converged solution. This inaccuracy is most pronounced for cases where the 


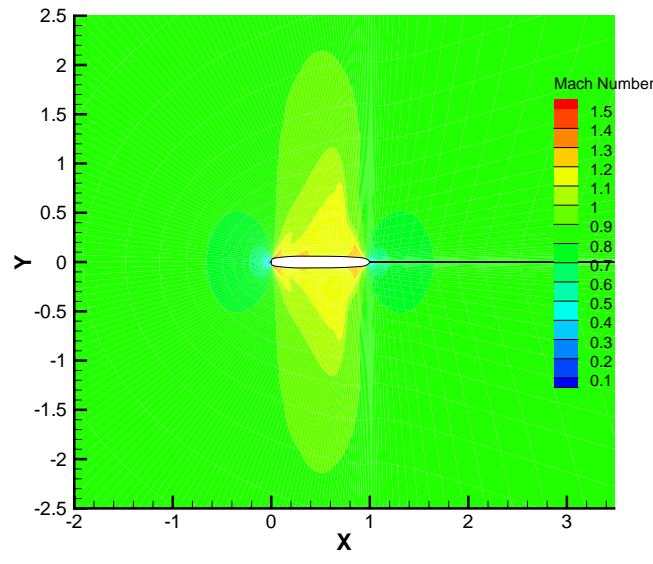

(a) No pressure switch

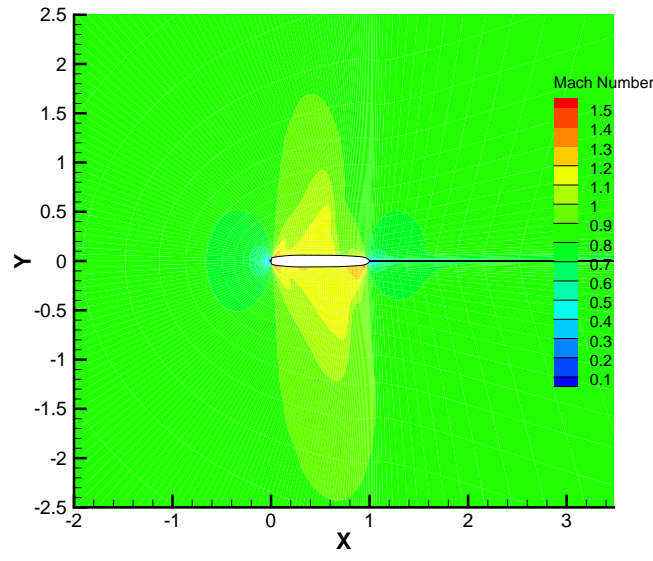

(b) Pressure switch

Figure 2.7: Comparison of Mach contours of NACA 0012 airfoil with and without pressure switch

final optimized geometry is not shock-free. For these cases, it is not possible to perform the optimization without the pressure switch, as the optimizer is receiving incorrect flow functionals. Therefore the only option is to include the pressure switch in the gradient. Osusky [19] looked at the effect of pressure switch linearization for viscous flows, and concluded the linearization was necessary for gradient accuracy.

\subsubsection{SNOPT Optimization}

The linear constraints, typically geometric constraints to group control points, can be satisfied exactly without performing a flow solve. Examples of nonlinear constraints are constant projected area, minimum wing volume and constant lift coefficient. The nonlinear constraints are satisfied to a user-specified tolerance of $10^{-6}$. For a typical aerodynamic shape optimization problem, the objective function is nonlinear, and the constraints imposed are a combination of linear and nonlinear constraints. For this type of problem, SNOPT applies a sparse sequential quadratic programming (SQP) method that uses a limited-memory quasi-Newton approximation to the Hessian.

The SQP algorithm requires both major and minor iterations. The major iterations generate a sequence of iterates that converge to the optimal solution. The solution of a quadratic programming (QP) subproblem is used to generate a search direction towards the next iterate, 
which is solved via minor iterations. It is in the solution of the QP subproblem where the impact of the number of degrees of freedom is seen. The QP subproblem employs a two-phase active-set algorithm that solves

$$
\begin{gathered}
\operatorname{minimize} f_{k}+g_{k}^{T}\left(x-x_{k}\right)+\frac{1}{2}\left(x-x_{k}\right)^{T} H_{k}\left(x-x_{k}\right) \\
\text { subject to } c_{k}+J_{k}\left(x-x_{k}\right) \geq 0
\end{gathered}
$$

where $f_{k}$ is the objective function, $g_{k}$ is the gradient of the objective function, and the vector $x$ contains the design variables. The matrix, $H_{k}$, is a quasi-Newton approximation to the Hessian of the Lagrangian, and is updated after each major iteration using BFGS. The nonlinear constraints and linear constraints are converted to equalities using slack variables. The nonlinear constraints are then linearized. The second equation represents this linearization of the nonlinear constraints, where the vector $c_{k}$ contains the nonlinear constraints, and the gradients of these constraints form the Jacobian, $J_{k}$. At each minor iteration the constraints, $c_{k}$, are partitioned into basic, superbasic and nonbasic variables:

$$
B x_{B}+S x_{S}+N x_{N}=b
$$

The nonbasic variables are frozen at their upper and lower bounds, and therefore made active and part of the working active set for the minor iteration. An inequality constraint is considered active at a solution if

$$
c_{i}(x)=0, i \in \mathrm{I}
$$

meaning the constraint is at its bound. A search direction is sought that moves the superbasic variables in a direction that will improve the objective function. The basic variables change to satisfy the constraint equation and the nonbasic variables remain the same. The search direction then satisfies:

$$
B p_{B}+S p_{S}=0
$$

The superbasic variables represent the number of degrees of freedom that remain after the 
constraints are satisfied. The search direction is computed using the reduced-Hessian and the reduced-gradient. The method used to calculate this depends on the number of superbasic variables. When no more improvement can be found in the objective function, a nonbasic variable is added to the superbasic variable set, decreasing the working active set. In contrast, the working active set is increased by one when the step size is too large such that it violates the bounds of a basic or superbasic variable.

An optimization is deemed successful, or fully converged, when SNOPT is able to satisfy the KKT conditions to within a specified tolerance. The nonlinear constraints must be satisfied to within a tolerance of $10^{-6}$, referred to as the feasibility tolerance. The gradient of the Lagrangian must also meet a tolerance, referred to as the optimality tolerance, which is user specified and can vary from problem to problem, but ranges from $10^{-5}$ to $10^{-7}$. 



\section{Chapter 3}

\section{Results}

\subsection{Case 1: Symmetrical Airfoil Optimization in Transonic In- viscid Flow}

This case involves drag minimization of the NACA 0012 airfoil in transonic, inviscid flow and is based on work done by Vassberg et al. 27] The number of control points used to parameterize the airfoil is varied to investigate the effect the parameterization has on the optimization. In a two-dimensional inviscid flow, the only source of drag, other than numerical error (including the effect of the finite distance to the far-field boundary), is wave drag. Hence if the shocks can be eliminated, the drag is strictly a consequence of numerical error.

\subsubsection{Optimization Problem}

The optimization problem can be summarized as

$$
\begin{aligned}
& \text { minimize } C_{\mathrm{D}} \\
& \text { wrt } z \\
& \text { subject to } z \geq z_{\text {baseline }}
\end{aligned}
$$

where $C_{\mathrm{D}}$ is the drag coefficient, $z$ is the vertical coordinate of the optimized geometry, and $z_{\text {baseline }}$ is the vertical coordinate of the baseline geometry. The geometry is subject to a min- 
imum thickness constraint which requires the optimized geometry to be greater in thickness than the baseline geometry. The thickness constraint is enforced by using 7 nonlinear thickness constraints at $15 \%, 20 \%, 22 \%, 24 \%, 26 \%, 29 \%$, and $35 \%$ chord, as done by Bisson, Nadarajah and Dong [3]. These locations are chosen because the optimizer search direction tries to reduce the airfoil thickness in this region. The thickness at other chordwise locations will be confirmed after the final shape is obtained to ensure the thickness constraint is not violated. The use of nonlinear constraints is preferred in place of linear constraints on the control points, as it is independent of the initial fit of the airfoil. Since the B-spline fit is not exact, the initial fit of the NACA 0012 airfoil can vary slightly based on the number of control points used. If the constraints are placed on the control points, rather than the surface itself, the optimization problem is changed slightly when the parameterization is changed. Using nonlinear thickness constraints that constrain the surface ensures that the optimization problem is identical regardless of the parameterization. Since the initial airfoil is symmetric, the resulting lift coefficient is zero, which should be maintained throughout the optimization. The symmetry is enforced by adding linear constraints to the control points on the upper and lower surfaces that force them to be equal and opposite in sign. The optimization is given an optimality tolerance of $10^{-7}$; however, not all cases are able to achieve this level of convergence. The feasibility tolerance for nonlinear constraints is $10^{-6}$.

\subsubsection{Flow Conditions}

The flow is inviscid and transonic with a freestream Mach number of $M=0.85$ and zero angle of attack $\left(\alpha=0^{\circ}\right)$.

\subsubsection{Initial Geometry}

The NACA 0012 airfoil is modified to have a zero thickness trailing edge. The modified airfoil is defined as

$$
z_{\text {baseline }}= \pm 0.6\left(0.2969 \sqrt{x}-0.1260 x-0.3516 x^{2}+0.2843 x^{3}-0.1036 x^{4}\right), \quad x \in[0,1],
$$




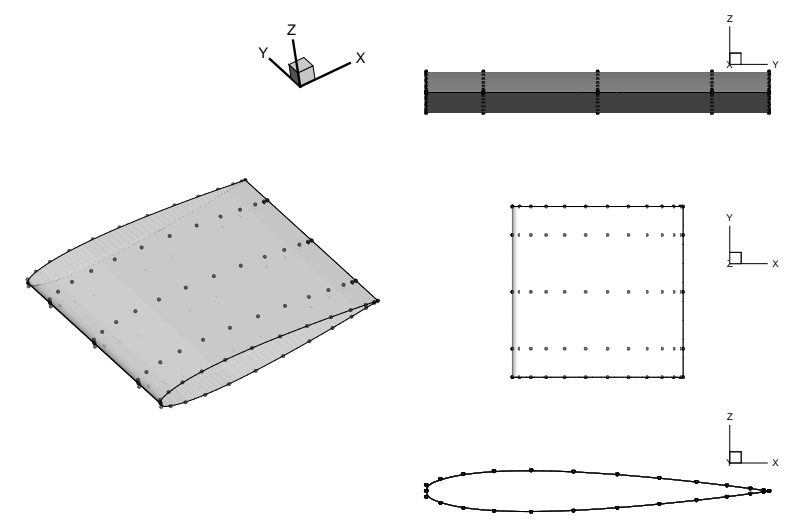

Figure 3.1: NACA 0012 extruded airfoil initial geometry

where the modification to the trailing edge occurs via a change in the $x^{4}$ term. In order to use the three-dimensional optimization algorithm, the optimization is performed using an extruded airfoil. The airfoil is extruded one chord length, so it has a chord of one unit and a span of one unit. The extruded airfoil is composed of an upper and lower patch. Each patch is parameterized using a variable number of control points in the streamwise direction and a constant number of control points (five) in the spanwise direction. Figure 3.1 shows the initial geometry parameterized with fourteen streamwise control points on each surface.

\subsubsection{Grid}

A structured grid is created around a flat plate with a chord length of one unit and a span of one unit. The mesh movement capabilities of the algorithm are then used to inflate this flat plate into an extruded airfoil with the sections determined from the B-spline fit of the NACA 0012 airfoil. Just as the geometry can be thought of as the extrusion of a two-dimensional airfoil, the grid can be thought of as an extrusion of a two-dimensional grid. There are ten nodes in the extruded direction, meaning the three-dimensional grid is ten times larger than its equivalent two-dimensional grid. Three different grid levels are created by starting with a fine grid and removing every other grid node, except in the extruded direction where the number of nodes remains constant. The fourth grid level (superfine) is created by refining the fine grid by doubling the number of nodes in the streamwise and offwall direction. Figure 3.2 shows the coarse grid level. 


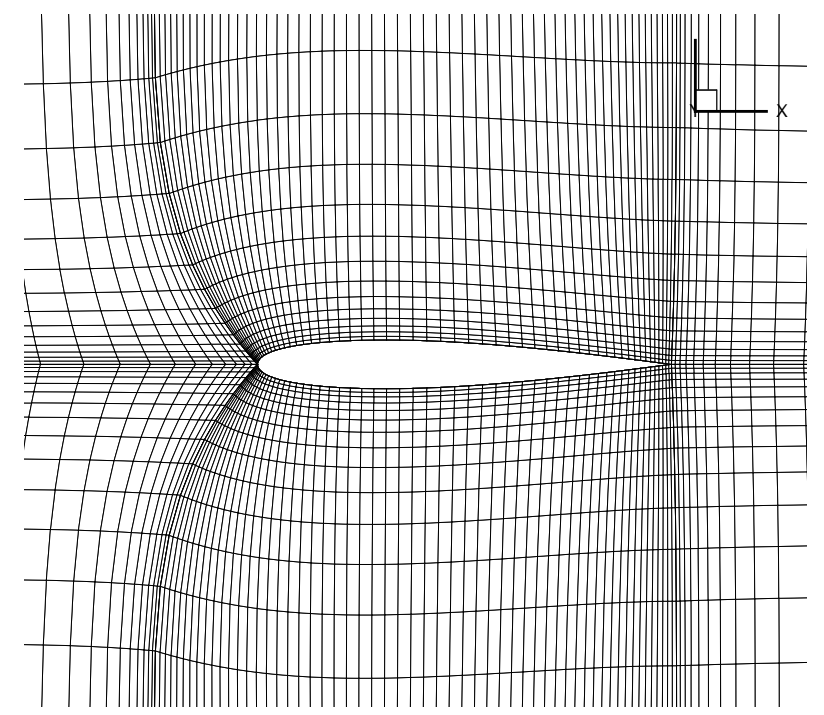

Figure 3.2: Coarse grid level for NACA 0012 extruded airfoil

\section{Grid Study - Initial Geometry}

Table 3.1 outlines the number of nodes, the streamwise spacing at the leading and trailing edges, and the off-wall spacing of the different grid levels. The number of nodes reported is the two-dimensional equivalent, calculated by taking the total number of nodes in the threedimensional grid and dividing by ten (the number of nodes in the extruded direction). A grid study of the initial geometry is conducted by performing a flow solve on each grid level. The fine grid is inflated using a parameterization with 48 streamwise control points on the upper and lower surfaces. A relatively high number of control points is chosen to ensure a highly accurate B-spline fit of the airfoil. The next two grid levels are created by subsequently removing every other node in the streamwise and offwall direction. The superfine grid level is created by refining the fine grid then splitting the blocks. Table 3.2 shows that the medium and fine grid levels are within 3/10 of a drag count of each other. The fine and superfine grid levels produce the same drag coefficient of 457.327 drag counts. Vassberg et al. [27] obtained an initial drag coefficient of 468.9 drag counts, while Bisson et al. [3] obtained an initial drag of 464.2 drag counts on their finest grid. Carrier et al. [5] obtained a zero mesh size drag coefficient of 471.1 counts. The initial drag coefficient obtained by Jetstream on the superfine grid is 7 counts below the lowest value obtained by Bisson et al. 
Table 3.1: Grid parameters for NACA 0012 airfoil grid study

\begin{tabular}{ccccc} 
Grid & Nodes (2D) & $\begin{array}{c}\text { Off-wall } \\
\text { Spacing }\end{array}$ & $\begin{array}{c}\text { Leading-Edge } \\
\text { Spacing }\end{array}$ & $\begin{array}{c}\text { Trailing-Edge } \\
\text { Spacing }\end{array}$ \\
\hline Coarse & 12760 & 0.008 & 0.008 & 0.008 \\
Medium & 49020 & 0.004 & 0.004 & 0.004 \\
Fine & 192100 & 0.002 & 0.002 & 0.002 \\
Superfine & 768400 & 0.001 & 0.001 & 0.001 \\
\hline
\end{tabular}

Table 3.2: Results of grid study for NACA 0012 airfoil inflated using 48 streamwise control points per patch

\begin{tabular}{ccc} 
Grid Level & Nodes (2D) & $\begin{array}{c}\text { Drag Coefficient } \\
\text { (Counts) }\end{array}$ \\
\hline Coarse & 12760 & 461.299 \\
Medium & 49020 & 457.598 \\
Fine & 192100 & 457.327 \\
Superfine & 768400 & 457.327 \\
\hline
\end{tabular}

\subsubsection{Optimization Results}

The results of the optimization using Jetstream can be compared to those obtained by Vassberg et al. [27] They used a Bezier curve geometry parameterization along with a response-surface optimization approach. Optimization using Jetstream is performed on the coarse, medium and fine grid levels. Figure 3.3 shows the results obtained performing optimization using Jetstream and compares them to those obtained by Vassberg et al. The number of control points used to parameterize each surface in the streamwise direction is varied from 5 to 13 . The leading and trailing edge control points are fixed, and the movement of the lower surface control points is constrained to be symmetric to the upper surface control points. The number of design variables for each case is then equal to the number of streamwise control points per surface, subtracting the two fixed control points. Therefore, the number of design variables is varied from 3 to 11. The drag coefficients obtained by Jetstream are significantly lower than those obtained by Vassberg et al., where the lowest drag coefficient obtained is 103.8 drag counts. One explanation for this could be grid density. The grid used by Vassberg et al. has 65,536 nodes, compared to the 2D-equivalent of 192,100 for the fine grid used with Jetstream. However, the medium grid, which has a similar grid density at 49,020 nodes, is also able to achieve lower drag values. Another explanation for the lower drag values is the parameterization used. The Bezier curve parameterization used by Vassberg et al. has very few design variables near the 


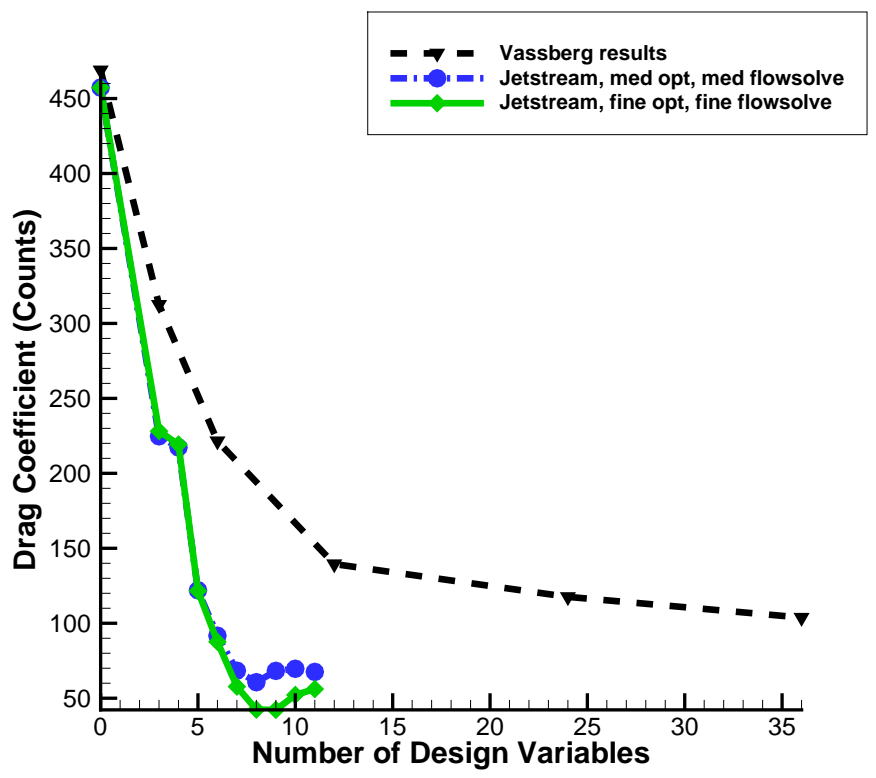

Figure 3.3: Comparison of optimization of the NACA 0012 airfoil using Jetstream on fine and medium grids with Vassberg et al. results [27]

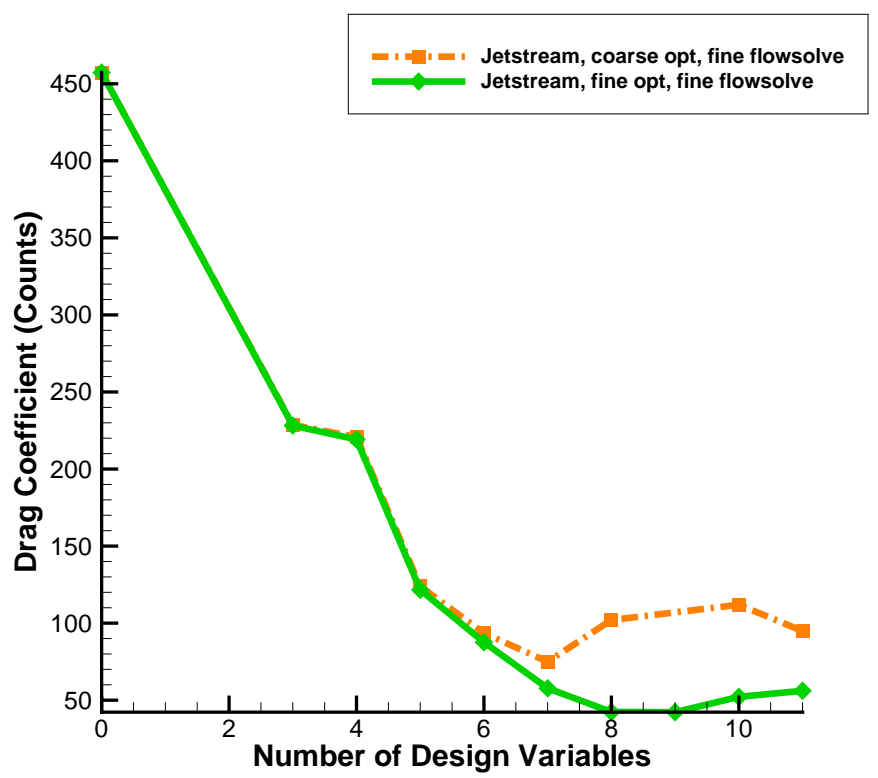

Figure 3.4: Comparison of Jetstream results on different grid levels for NACA 0012 airfoil 


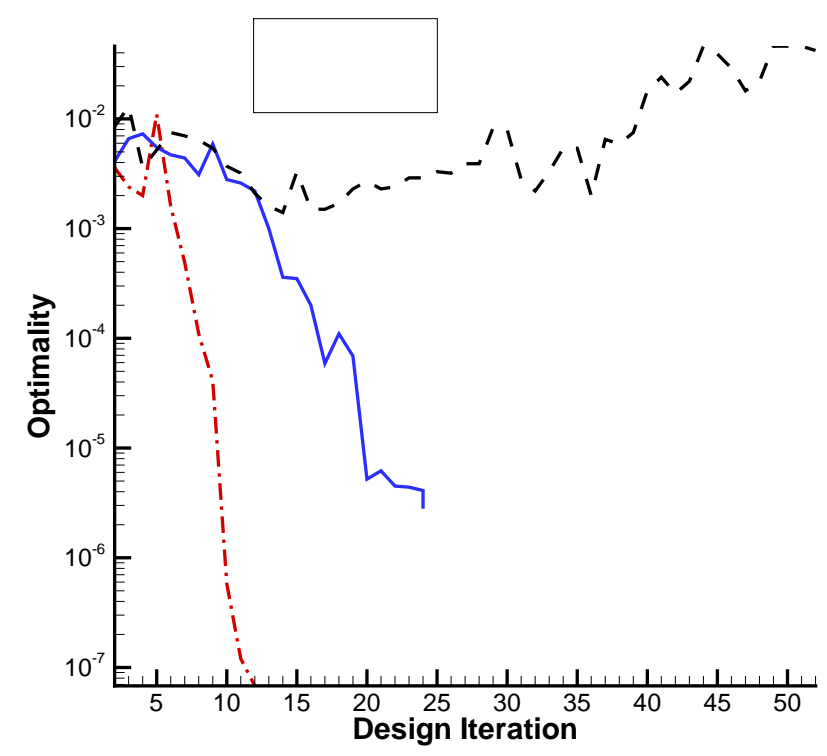

Figure 3.5: Typical convergence histories for NACA 0012 airfoil optimization

trailing edge, whereas the B-spline curve parameterization has more design variables in this region. The B-spline parameterization has roughly half of the design variables in the first $50 \%$ of chord length measured from the leading edge, and half in the second 50\%. With the Bezier parameterization, $5 / 6$ of the design variables are within the first $50 \%$ of chord length, and $1 / 6$ of the design variables are in the second $50 \%$. Since the majority of the geometry change is occurring near the trailing edge, the B-spline parameterization is better suited than the Bezier parameterization, as shown by Carrier et al. [5] Looking at the optimization results on the fine grid, as the number of design variables increases, the drag obtained decreases. The lowest drag coefficient is obtained on the fine grid level with 9 design variables: 42.24 drag counts, which is a reduction of $91 \%$ relative to the baseline geometry. However, after 9 design variables, the drag increases slightly. Figure 3.4 compares the results of optimization using the coarse grid (with a flowsolve on the final geometry using the fine grid) to optimization using the fine grid. In all cases the final drag obtained on the fine grid is lower than that obtained on the coarse grid. Using 3, 4, and 5 design variables, this difference is 2 drag counts or less. With 6 design variables the fine grid optimization produces a geometry almost 6 drag counts lower than that from the coarse grid, and with 8 design variables the difference is approximately 60 drag counts.

As the number of design variables increases, the convergence of the optimizer is adversely 
affected. Figure 3.5 shows the optimization history for three cases. With a low number of design variables, the optimality tolerance of $10^{-7}$ is achieved. As the number of design variables increases, the optimality tolerance is no longer met and only a one order of magnitude reduction in optimality is achieved. Figure 3.6 shows the airfoil shapes produced for three different parameterizations. As the number of control points is increased, the airfoil becomes thicker near the trailing edge. In addition, the thickness increases near the leading edge. The optimizer maintains the original thickness of the airfoil at approximately $25 \%$ chord. Figure 3.7 shows the distribution of the surface pressure coefficient for the same three parameterizations. The shock increases slightly in strength but is pushed further downstream. Figures 3.8 and 3.9 show the Mach and entropy contours for the initial NACA 0012 airfoil and the optimized shape with 9 design variables. The shock is not completely eliminated, but now only extends a small distance into the flow. 


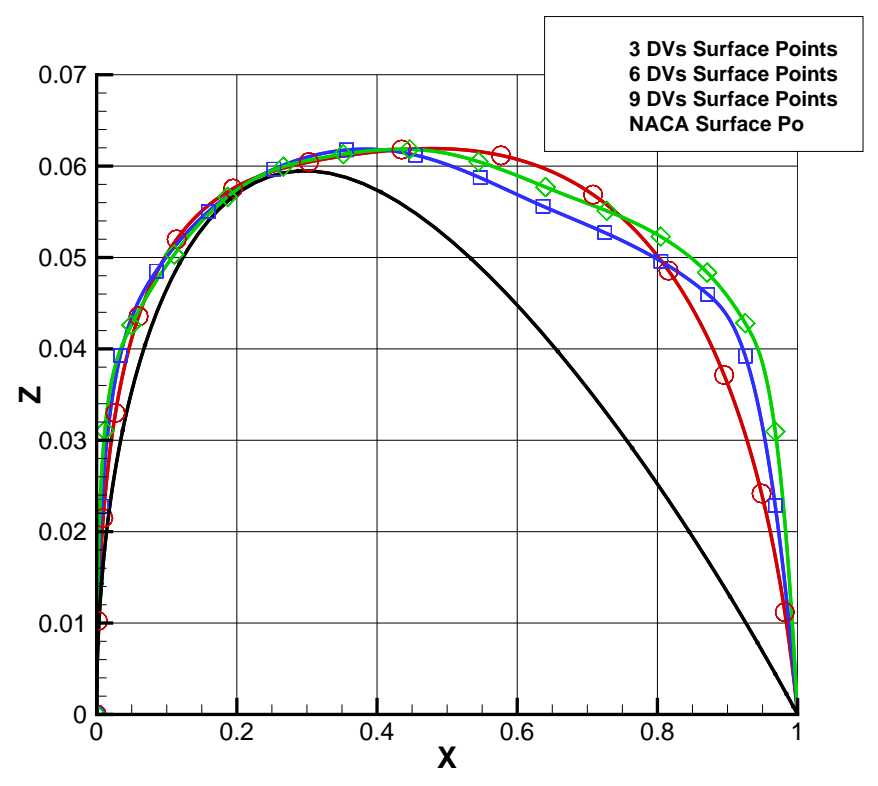

Figure 3.6: Comparison of final optimized airfoil shapes to initial NACA 0012 airfoil

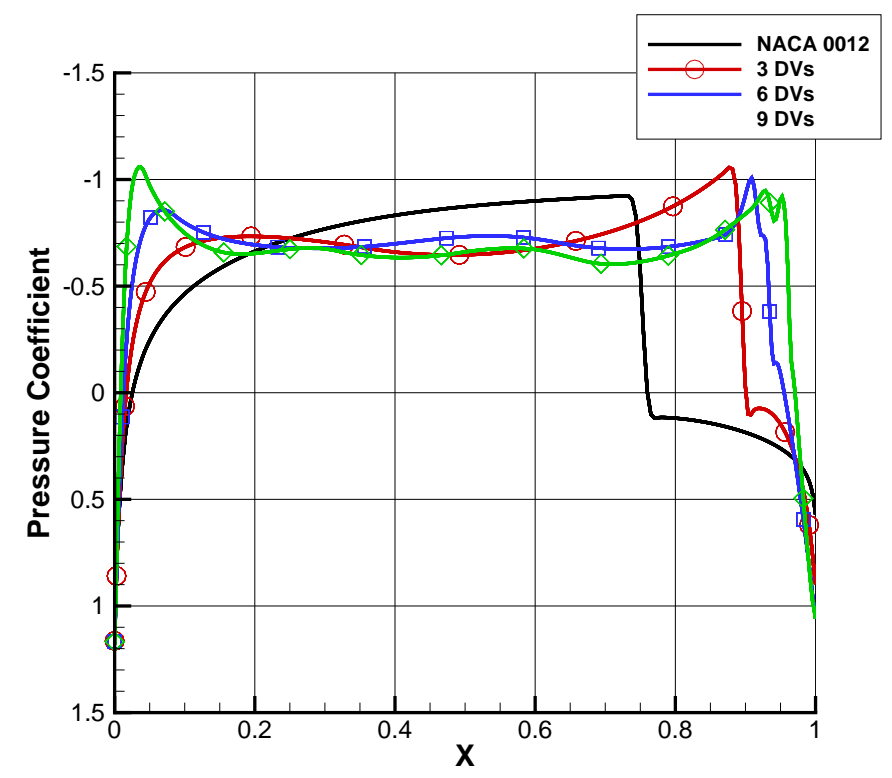

Figure 3.7: Comparison of final coefficient of pressure distributions to initial NACA 0012 airfoil 


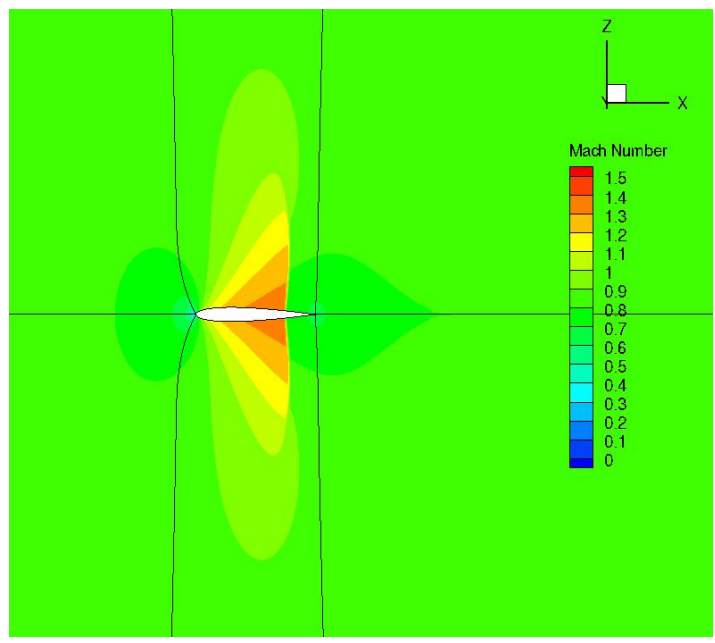

(a) Initial NACA 0012 Airfoil

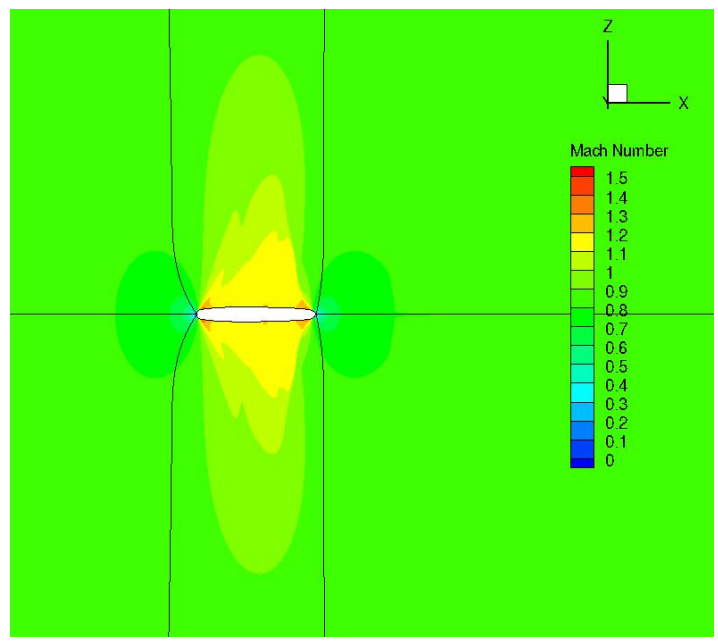

(b) Final, 9 Design Variables

Figure 3.8: Comparison of Mach contours for initial NACA 0012 airfoil and final optimized shape with 9 design variables

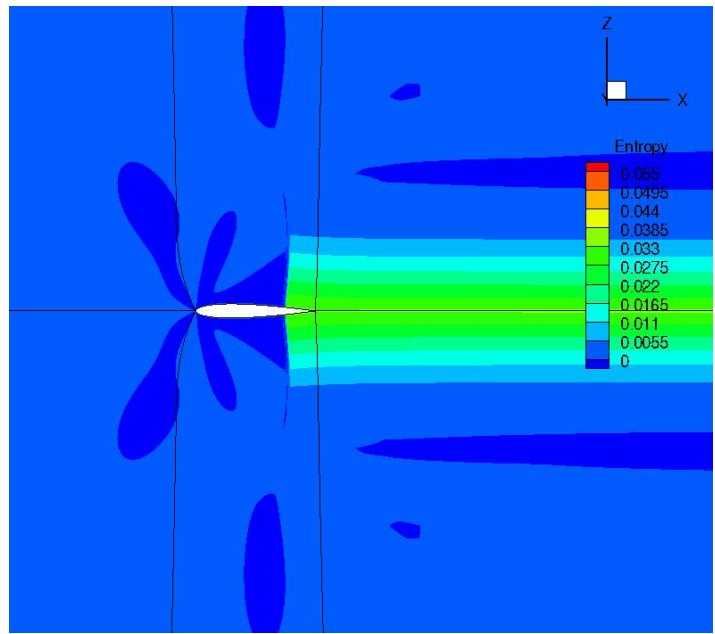

(a) Initial NACA 0012 Airfoil

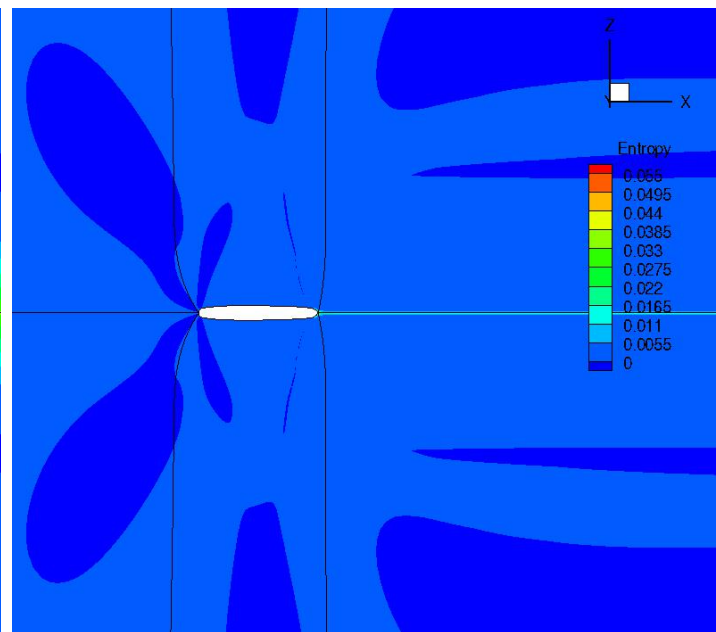

(b) Final, 9 Design Variables

Figure 3.9: Comparison of entropy contours for initial NACA 0012 airfoil and final optimized shape with 9 design variables 


\section{Grid Study - Final Geometry}

Tables 3.3 to 3.5 show the results of a grid study on the final geometries for a variety of parameterizations. Each table is based on the final optimized geometries using the coarse, medium and fine grids, respectively. The final geometries are analyzed by performing a flow solve on each grid level. For example, in Table 3.3 , the optimization is performed on the coarse grid, and then the final shape is analyzed using the medium and the fine grid. Unlike the grid study for the initial geometry, where there is a difference of less than 1 drag count between the medium and fine grids, the final geometries show a larger difference between the medium and fine grid levels. For the optimizations run using the medium grid, at higher parameterizations the flow solve either fails to converge on the coarse or fine grid level, or produces a solution with a non-zero lift coefficient. The failed flow solves only occur on the fine grid level, suggesting that the fine grid is resolving difficult flow features that were not present on the coarser grid levels. Given the presence of non-unique solutions for this case, it is likely that the flow is unsteady, but further analysis is required. For the fine optimization, fine flow solve case with 10 design variables, the order of accuracy is calculated to be $p=2.46$, and the grid converged value of drag is $C_{\mathrm{D}}^{*}=46.4$ drag counts. Comparing to the grid converged value of drag for the initial geometry, $C_{\mathrm{D}}^{*}=457.3 \mathrm{drag}$ counts, this is a reduction of $90 \%$. A grid converged value of

drag for the best case, with 9 design variables, could not be calculated due to the non-unique solution obtained on the medium grid. 
Table 3.3: Grid study for NACA 0012 airfoil optimization on coarse grid

Drag Coefficient, $C_{\mathrm{D}}$ (Counts)

\begin{tabular}{cccc} 
Design Variables & Coarse Flowsolve & Medium Flowsolve & Fine Flowsolve \\
\hline 3 & 221.34 & 224.99 & 228.61 \\
4 & 217.31 & 218.72 & 220.83 \\
5 & 145.93 & 124.05 & 123.8 \\
6 & 138.87 & 96.53 & 93.61 \\
7 & 143.23 & 82.48 & 74.93 \\
8 & 151.87 & 108.21 & 102.03 \\
9 & 149.98 & 116.74 & FAILED \\
10 & 143.64 & 113.41 & 111.96 \\
11 & 136.17 & 97.46 & 94.88 \\
\hline
\end{tabular}

Table 3.4: Grid study for NACA 0012 airfoil optimization on medium grid Drag Coefficient, $C_{\mathrm{D}}$ (Counts)

\begin{tabular}{cccc} 
Design Variables & Coarse Flowsolve & Medium Flowsolve & Fine Flowsolve \\
\hline 3 & 221.09 & 224.72 & 228.33 \\
4 & 216.61 & 217.28 & 219.3 \\
5 & 146.88 & 122 & 121.71 \\
6 & 152.55 & 91.71 & 87.82 \\
7 & 168.94 & 68.36 & NON ZERO LIFT \\
8 & NON ZERO LIFT & 60.67 & NON ZERO LIFT \\
9 & 200.39 & 68.25 & FAILED \\
10 & NON ZERO LIFT & 69.64 & NON ZERO LIFT \\
11 & NON ZERO LIFT & 67.56 & FAILED \\
\hline
\end{tabular}

Table 3.5: Grid study for NACA 0012 airfoil optimization on fine grid Drag Coefficient, $C_{\mathrm{D}}$ (Counts)

\begin{tabular}{cccc} 
Design Variables & Coarse Flowsolve & Medium Flowsolve & Fine Flowsolve \\
\hline 3 & 220.96 & 224.58 & 228.2 \\
4 & 216.68 & 217.23 & 219.22 \\
5 & 146.42 & 121.96 & 121.62 \\
6 & 143.77 & 91.86 & 87.63 \\
7 & 169.32 & 68.86 & 57.74 \\
8 & NON ZERO LIFT & 58.99 & 42.39 \\
9 & 229.38 & NON ZERO LIFT & 42.24 \\
10 & 219.25 & 77.93 & 52.18 \\
11 & 244.86 & 87.14 & 56.1 \\
\hline
\end{tabular}




\subsection{Case 2: Twist Optimization of a Rectangular Wing in Sub- sonic Inviscid Flow}

The second case investigated is the drag minimization of a rectangular wing with NACA 0012 sections through optimization of the twist distribution about the trailing edge. The number of design variables is varied to investigate the effect on the optimization. The flow is subsonic and inviscid; hence the goal is to minimize the induced drag at a fixed lift coefficient. The optimization should recover a lift distribution that is close to elliptical and a span efficiency factor close to one. The span efficiency factor is calculated using

$$
e=\frac{C_{\mathrm{L}}^{2}}{\pi \Lambda C_{\mathrm{D}_{\mathrm{i}}}}
$$

where $e$ is the span efficiency factor, $C_{\mathrm{L}}$ is the lift coefficient, $\Lambda$ is the aspect ratio and $C_{\mathrm{D}_{\mathrm{i}}}$ is the induced drag coefficient.

\subsubsection{Optimization Problem}

The optimization problem is formally described as

$$
\begin{gathered}
\text { minimize } C_{\mathrm{D}} \\
\text { wrt twist } \\
\text { subject to } C_{\mathrm{L}}=0.375
\end{gathered}
$$

where $C_{\mathrm{D}}$ is the drag coefficient and $C_{\mathrm{L}}$ is the lift coefficient. The wing is twisted about the trailing edge at a finite number of spanwise stations through the use of linear constraints. This is accomplished by varying the $z$ coordinates, so the projected surface area remains constant. The span and aspect ratio are also fixed, so the only mechanism to reduce the induced drag coefficient is through the span efficiency factor, which is related to the spanwise load distribution. The tip is constrained to be a linear shear of the twist of the last two spanwise stations. This prevents the optimizer from twisting the tip so drastically that it creates a winglet. The lift constraint is the only nonlinear constraint implemented. The angle of attack is a design variable, and the 


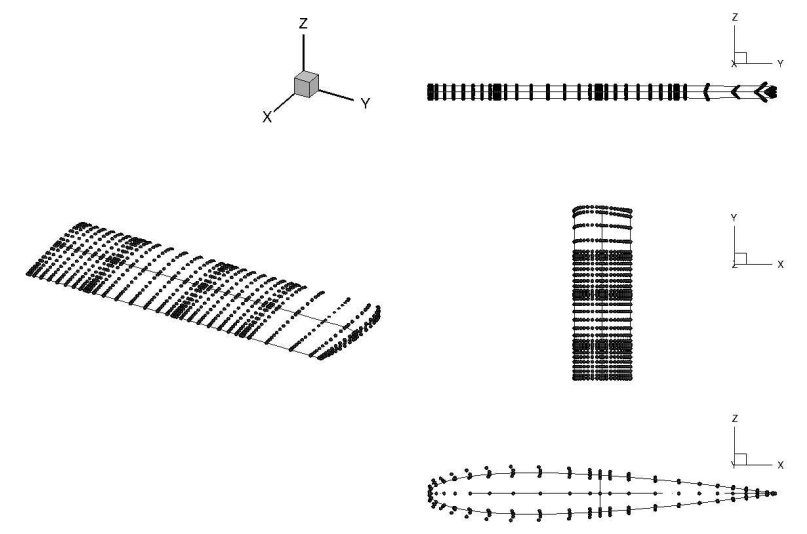

Figure 3.10: NACA 0012 rectangular wing initial geometry

root section is fixed. The optimization is given an optimality tolerance of $10^{-6}$; however, as in the first case, not all cases are able to achieve this level of convergence. The feasibility tolerance is $10^{-6}$.

\subsubsection{Flow Conditions}

The flow is invisicid and subsonic. The freestream Mach number is $M=0.5$, and the initial angle of attack is $\alpha=4.4^{\circ}$.

\subsubsection{Initial Geometry}

The geometry is a rectangular wing with NACA 0012 sections which have been modified to have a sharp trailing edge (see Equation 3.1). The wing has a semispan of 3.06 chords, consisting of 3 chords of rectangular planform and 0.06 chords of wing-tip cap. The geometry has sixteen surface patches, twelve inboard (upper and lower) that have a variable spanwise parameterization and four outboard (upper and lower) that have a constant spanwise parameterization to ensure a consistent tip geometry. Figure 3.10 shows the geometry with 13 streamwise and 11 spanwise control points on each inboard patch.

\subsubsection{Grid}

The grid has approximately 1.4 million nodes. The off-wall spacing is 0.002 chords, the streamwise leading-edge spacing is 0.005 chords, and the streamwise trailing-edge spacing is 0.01 


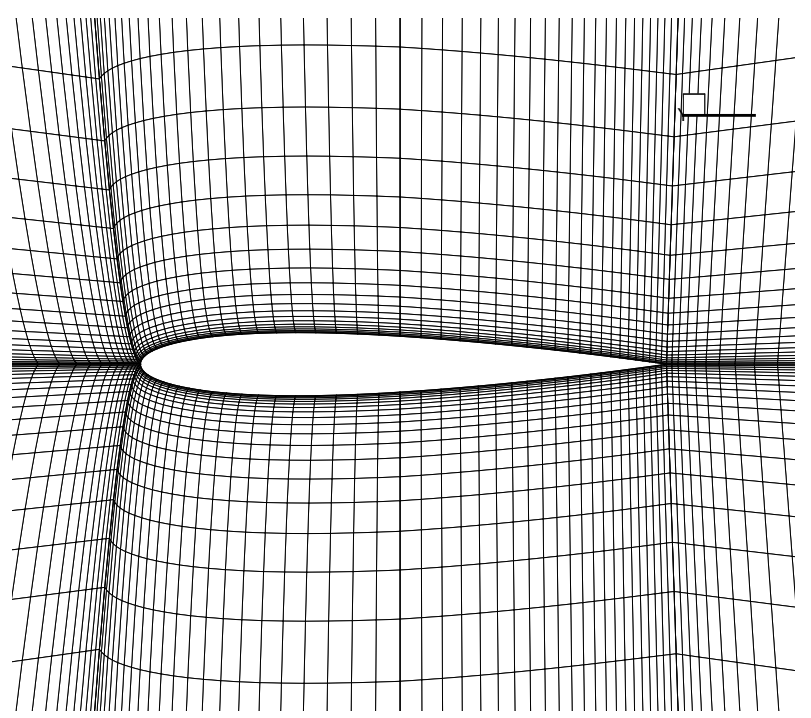

Figure 3.11: Grid for NACA 0012 rectangular wing at root

Table 3.6: Coefficients for rectangular wing with NACA 0012 sections on optimization mesh and fine mesh

\begin{tabular}{ccccc} 
& \multicolumn{2}{c}{ Initial Geometry } & \multicolumn{2}{c}{ Optimized Geometry } \\
\cline { 2 - 5 } Mesh Size (nodes) & $1.4 \times 10^{6}$ & $11.2 \times 10^{6}$ & $1.4 \times 10^{6}$ & $11.2 \times 10^{6}$ \\
\hline$C_{\mathrm{L}}$ & 0.375 & 0.375 & 0.375 & 0.375 \\
$C_{\mathrm{D}}$ & 0.00784 & 0.00773 & 0.00761 & 0.00759 \\
\hline
\end{tabular}

chords. Figure 3.11 shows a close-up of the node distribution around the grid at the root.

\section{Grid Study}

A finer grid is created by doubling the number of nodes in each direction, and then splitting the blocks to ensure a reasonable number of nodes per processor. This grid has approximately 10.2 million nodes, with an off-wall spacing of 0.001 chords, a streamwise leading-edge spacing of 0.0025 chords and a streamwise trailing-edge spacing of 0.005 chords. Table 3.6 shows the results of analyzing the initial and final geometry on the finer grid. The final geometry is from the best case studied, which used 13 streamwise and 11 spanwise control points on each inboard patch. On the coarse grid, the drag coefficient is reduced 2.3 drag counts from initial to final, which is $3 \%$ of the initial drag. On the fine grid, the drag coefficient is reduced 1.4 drag counts from initial to final, which is $1.8 \%$ of the initial drag. This shows that the improvement in drag coefficient carries over to finer grid levels. 


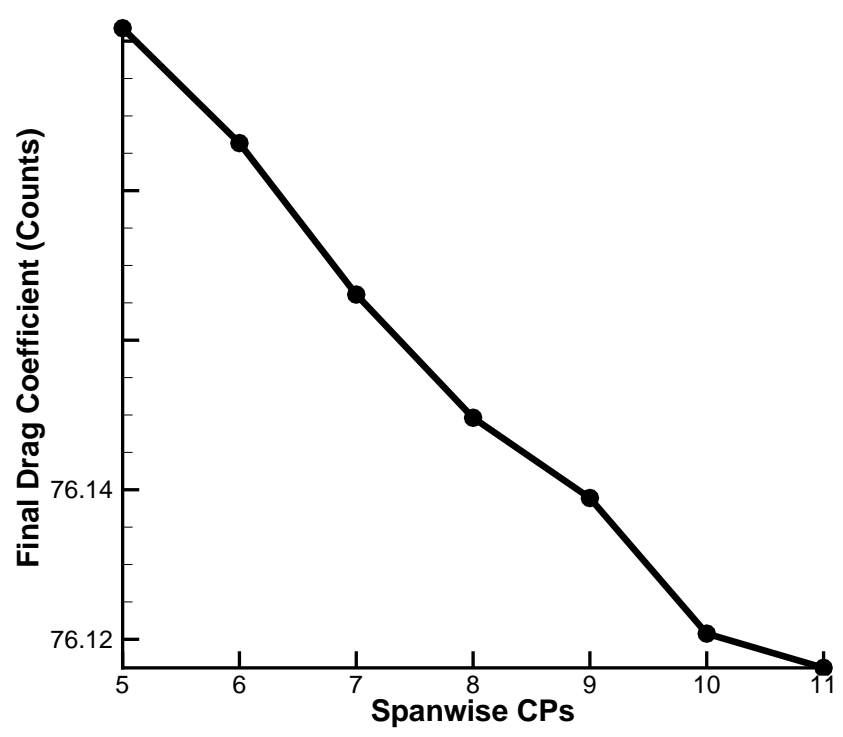

Figure 3.12: Effect of change in number of spanwise control points per inboard patch on final drag coefficient for NACA 0012 rectangular wing twist optimization case

\subsubsection{Optimization Results}

Figure 3.12 shows how the final drag coefficient changes as the number of spanwise control points per inboard patch increases. The number of spanwise control points is varied from 5 to 11, and as the number increases, the final drag obtained decreases. The drag coefficient for the untwisted wing is 78.4 drag counts, and the drag coefficient for the final optimized geometry with 11 spanwise control points is 76.1 drag counts, which is a reduction of $3 \%$. The difference between the coarsest parameterization with 5 control points and the finest parameterization with 11 control points is 0.09 drag counts. Figures 3.13 and 3.14 show how the span efficiency factor changes as the number of spanwise control points increases. The initial spanwise efficiency factor for the untwisted wing is 0.951 , and the final span efficiency factor varies from 0.979 with 5 spanwise control points to 0.980 with 11 spanwise control points. The increase in span efficiency is $3 \%$ from initial to final.

Figure 3.15 shows the optimization convergence history for two different parameterizations. Both have very similar convergence histories, and the optimality is decreased four orders of magnitude to reach the optimality tolerance of $10^{-6}$. All cases were able to achieve this required tolerance. Figure 3.16 shows the lift distribution for the case with 11 spanwise control points. 


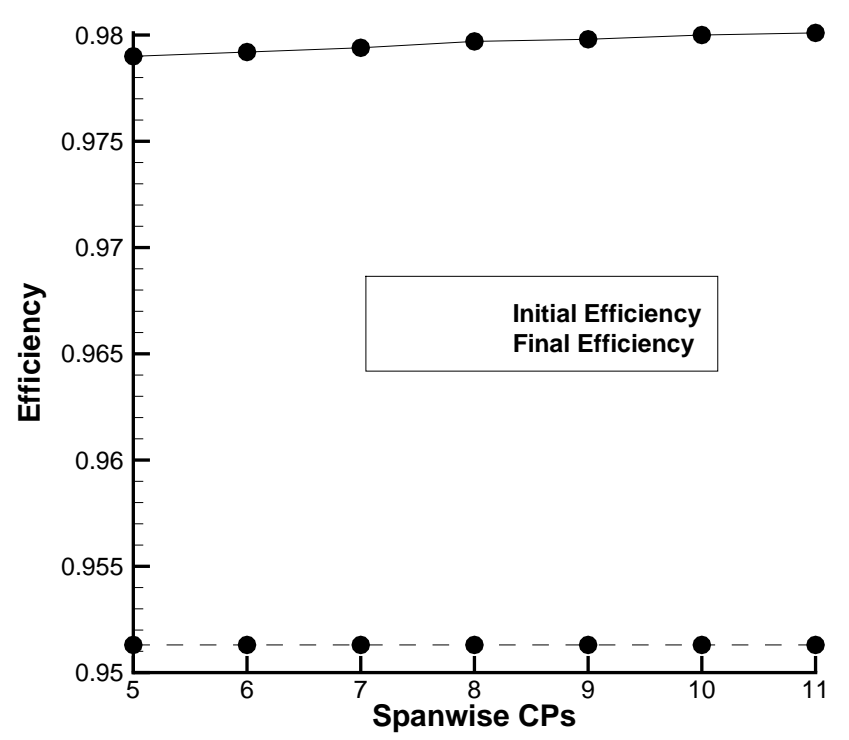

Figure 3.13: Effect of change in number of spanwise control points per inboard patch on final span efficiency factor for NACA 0012 rectangular wing twist optimization case

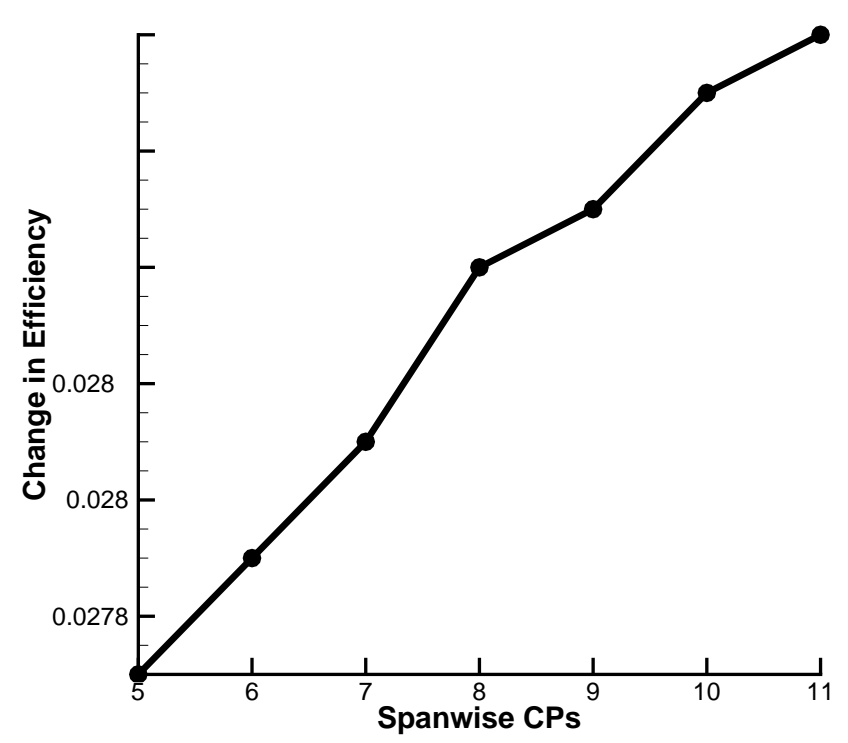

Figure 3.14: Effect of change in number of spanwise control points per inboard patch on change in span efficiency factor for NACA 0012 rectangular wing twist optimization case 


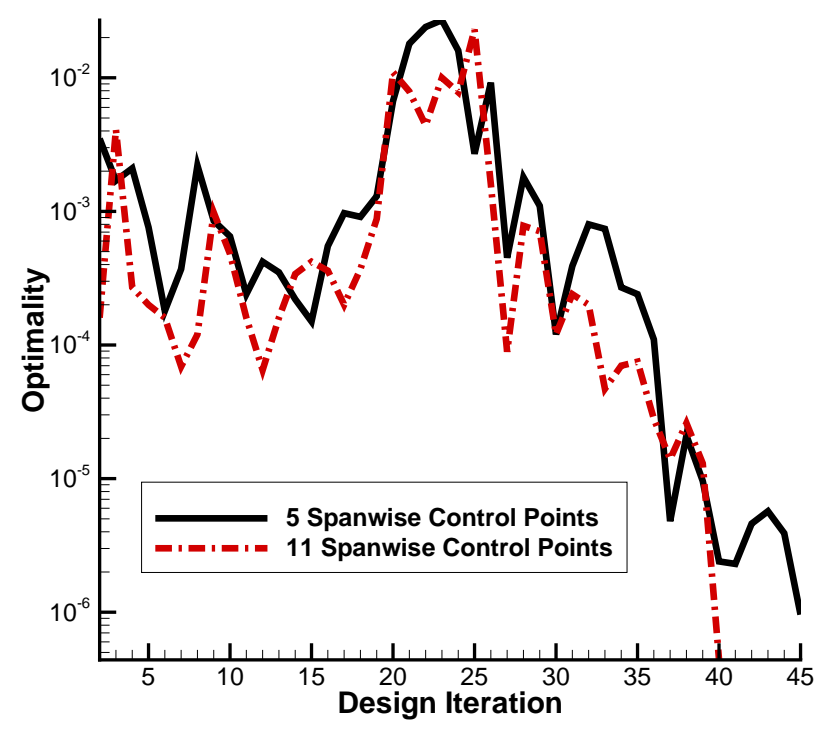

Figure 3.15: Typical optimization convergence history for NACA 0012 rectangular wing twist optimization case

The initial lift distribution, shown in blue, is far from elliptical. The final lift distribution is very close to elliptical, showing that the optimizer is able to produce a twist distribution that has reduced the induced drag. Figure 3.17 shows the twist distribution for the cases with 5 and 11 spanwise control points. The twist distributions are very similar, with the exception of the twist at the root, with the coarser parameterization producing a higher twist angle than the finer parameterization. The twist distribution is not continuous near the wing root, with the twist sharply jumping 3 degrees from $0 \%$ to $20 \%$ span. From $20 \%$ span to wing tip, the twist decreases smoothly. A smooth twist distribution throughout could be enforced by implementing additional constraints. 


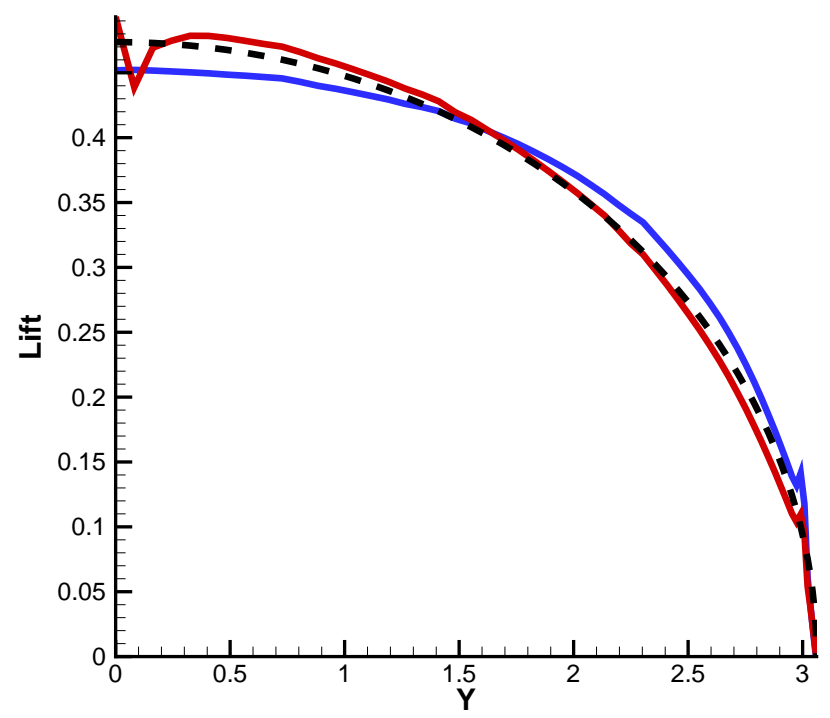

Figure 3.16: Lift distribution for NACA 0012 rectangular wing twist optimization case with 11 spanwise control points per inboard patch

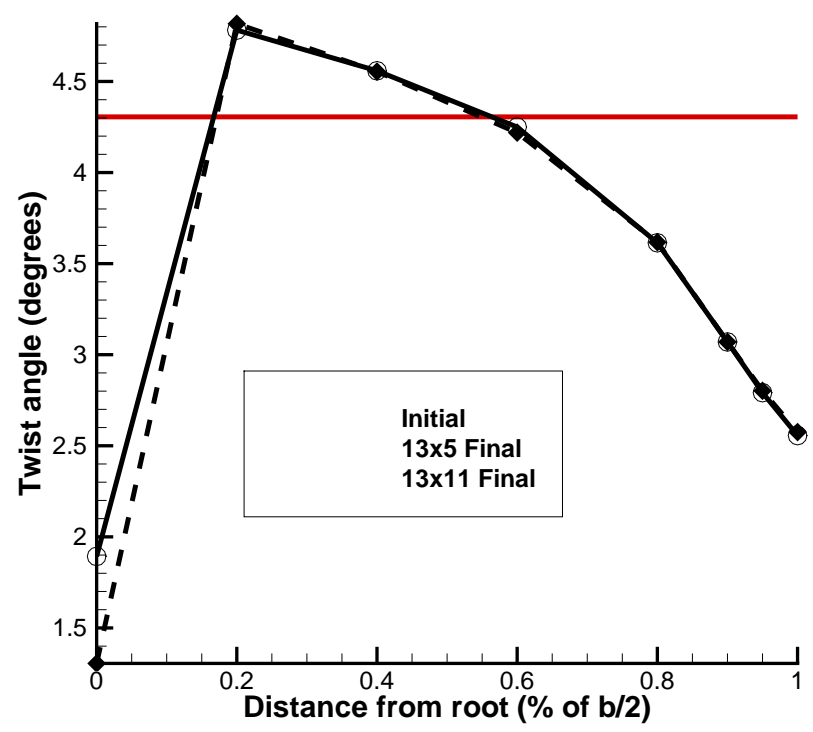

Figure 3.17: Comparison of twist distribution for cases with 5 and 11 spanwise control points per inboard patch to initial twist of NACA 0012 rectangular wing 


\subsection{Case 3: Wing Twist and Section Optimization in Transonic Turbulent Flow}

The final case is the lift-constrained drag minimization of the Common Research Model (CRM)

wing-only geometry in fully turbulent transonic flow. The $z$-coordinates of the control points are design variables, allowing for both twist and section shape optimization. Since this flow is viscous and transonic, all three types of drag are captured by the flow solver: viscous drag, lift-induced drag, and wave drag.

\subsubsection{Optimization Problem}

The optimization problem is

$$
\begin{aligned}
& \text { minimize } C_{\mathrm{D}} \\
& \text { wrt twist and section shape } \\
& \text { subject to } C_{\mathrm{L}}=0.5 \\
& \qquad C_{\mathrm{M}} \geq-0.17
\end{aligned}
$$

where $C_{\mathrm{D}}$ is the drag coefficient, $C_{\mathrm{L}}$ is the lift coefficient, and $C_{\mathrm{M}}$ is the pitching moment coefficient. The planform area, which is fixed throughout the optimization, is 3.407014 squared reference units, with the mean aerodynamic chord (MAC) used as the reference length. Both section changes and twist are permitted. This is achieved by allowing control point movement in the vertical $(z)$ direction and fixing the trailing edge. The planform area is fixed, and the angle of attack is a design variable. The lift coefficient is constrained at $C_{\mathrm{L}}=0.5$, and the pitching moment coefficient is constrained at $C_{\mathrm{M}} \geq-0.17$. There is a volume constraint such that the total wing volume is greater than or equal to its initial value. The thickness of the sections must be greater than or equal to $25 \%$ of the initial thickness.

\subsubsection{Flow Conditions}

The flow is viscous, transonic, and fully turbulent. The Mach number is 0.85 , Reynolds number 5 million, and the initial angle of attack 2.2 degrees. The pitching moment is taken about the 


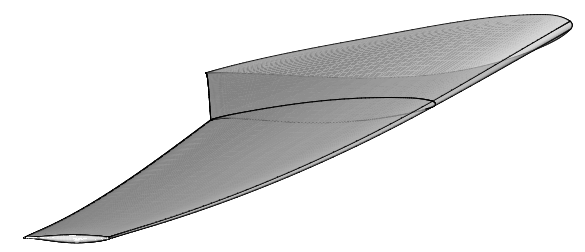

Figure 3.18: Initial CRM wing geometry

point $(1.2077,0,0.00769)$.

\subsubsection{Initial Geometry}

The baseline geometry is the extracted wing from the Common Research Model, which was used in the Fourth and Fifth Drag Prediction Workshops [28, 17]. The geometry, shown in Figure 3.18, features a blunt trailing edge. The wing geometry is extracted by deleting the fuselage from the wing-body geometry, leaving the wing root located at a distance of 120.52 inches from the original symmetry plane. The leading edge of the wing root is translated to the origin, and all grid coordinates are scaled by the MAC of 275.8 inches. A post-processing script is applied to all nodes on the symmetry plane to ensure that they are at $y=0$.

\subsubsection{Grid}

Two different blocking structures are used on an identical mesh: one with fewer blocks for geometry parameterization and one with more blocks for the flow solve. An O-O topology computational mesh made up of 18 blocks and 3.38 million nodes is used for the parameterization of the geometry. Flow analysis is performed on a mesh that is created by subdividing each block of the parameterization mesh into 8 sub-blocks, resulting in a 144-block, 3.57 million-node mesh, enabling perfect load balancing on 144 processors. The mesh has an off-wall spacing of $2.56 \times 10^{-6}$ reference units, resulting in an average $y^{+}$value of 1.0 . The wing geometry has 9 surface patches: 2 each on the upper and lower surfaces, 2 each along the leading edge and blunt 
trailing edge, and one cap patch at the wing tip. The leading-edge patches (one for the inboard section and one for the outboard) are required in order to match with the blunt trailing-edge patches, and also serve as a method of better capturing the curvature of the leading edge.

In her Ph.D. thesis, Osusky [19] analyzed the initial and optimized geometries on a finer mesh made up of $39.4 \times 10^{6}$ nodes and compared the results to those obtained on the $3.6 \times 10^{6}$ node mesh. When the analysis is done on the fine mesh, the pitching moment constraint is violated, however the difference is less than $2 \%$. The drag coefficients are $4-7 \%$ lower on the fine mesh as compared to the coarse mesh. This analysis shows that the final geometry produced reduces the drag relative to the initial geometry, even on a refined mesh.

\subsubsection{Optimization Results}

As in previous cases, the number of design variables used to parameterize the geometry is varied to determine the effect this has on the final result. Figure 3.19 shows how the final drag coefficient changes with the number of design variables. The data points represented by red circles are parameterized using 5 spanwise control points on each surface patch, and then the number of streamwise control points per patch is increased from 5 to 9 . The data points represented by blue squares are parameterized using 6 spanwise control points, with the same pattern applied to the streamwise control points. The black triangle data points have 7 spanwise control points. The lowest drag coefficient, 192.63 drag counts, is obtained with the coarsest streamwise parameterization (5 control points per patch) and finest spanwise parameterization (7 control points per patch). This is almost 20 drag counts lower than the initial drag coefficient of 212 drag counts, which is a $9 \%$ reduction in drag. The drag coefficient decreases as the number of spanwise control points used increases. As the number of spanwise control points increases, there is more flexibility for the geometry to achieve an improved twist distribution. The drag coefficient generally increases as the number of streamwise control points used increases. This is contrary to the results seen by Carrier et al. [5], however their use of camber and twist modifications, not section shape and twist modifications, means the problem was constrained differently. The thickness constraint that requires the section shapes to maintain at least $25 \%$ of the original thickness is imposed on the control points, not the surface itself. This means that the thickness constraints vary slightly depending on the initial parameterization. It would 


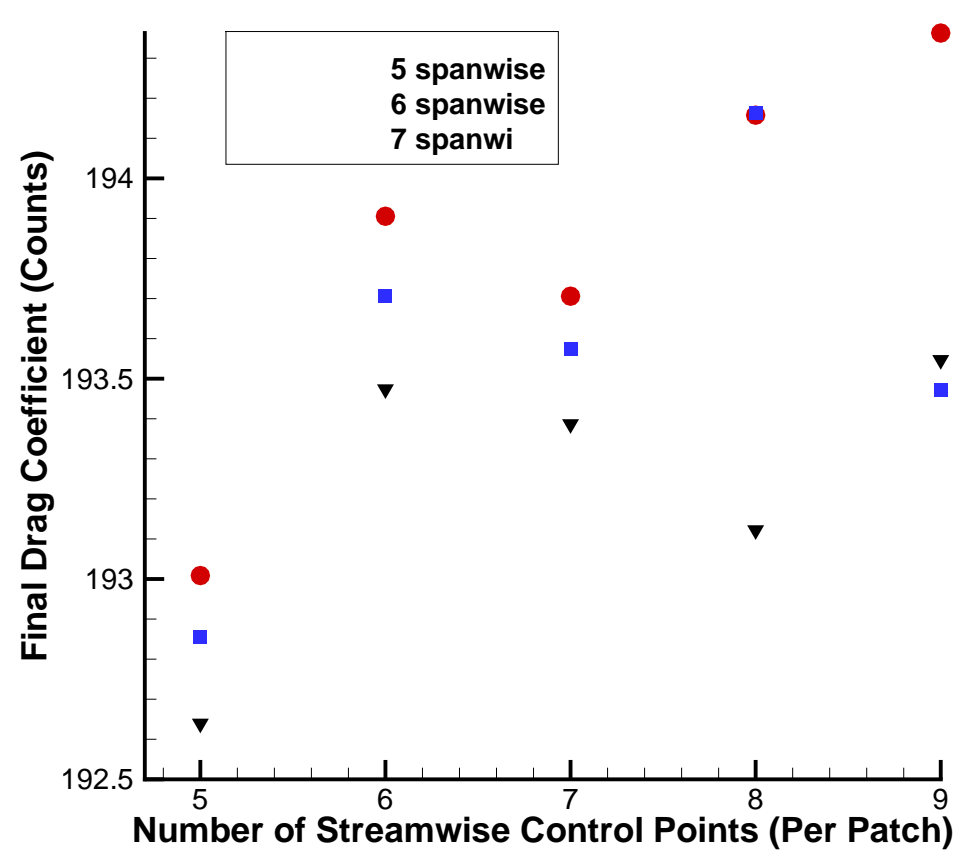

Figure 3.19: CRM wing drag results - all parameterizations

be preferable to use nonlinear thickness constraints that enforce the thickness between surface points, not between control points.

It is important to note that the optimizer is not fully converging for any of the cases. An example of a typical convergence history is shown in Figure 3.20 for two cases: 5 streamwise and 5 spanwise control points per patch, and 5 streamwise and 7 spanwise control points per patch. The optimality is reduced by roughly an order of magnitude overall, with the optimizer unable to achieve deep convergence. With the number of surface patches required to fit this geometry, the number of design variables ranges from 150 - 300, depending on the parameterization. The high number of design variables is making it difficult for the optimizer to fully converge.

Figure 3.21 compares the initial and final lift distribution for the case with 5 streamwise and 7 spanwise control points. The final distribution is closer to elliptical than the initial distribution, showing that the optimizer has reduced the induced drag. Surface pressure coefficient distributions and airfoil section shapes are shown in Figures 3.22 and 3.23 , respectively. These compare the initial and final results for the case with 5 streamwise and 7 spanwise control points. The pressure distributions show that the optimizer has changed the geometry to 


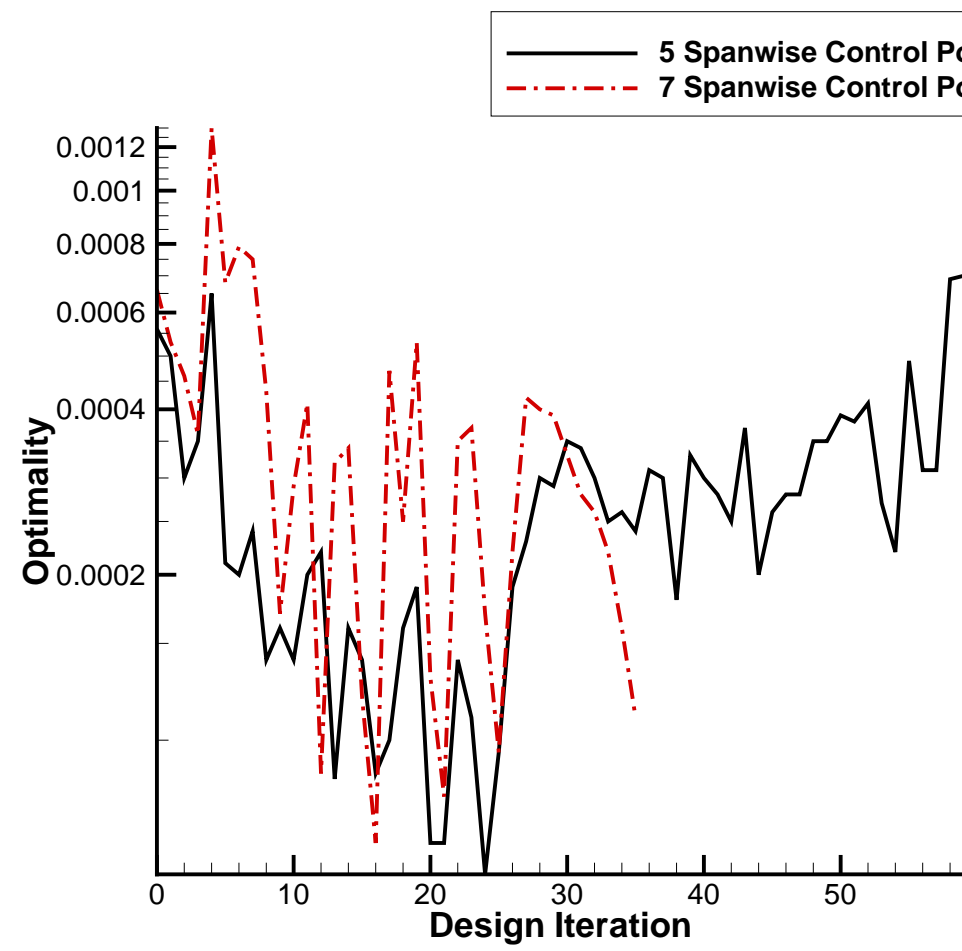

Figure 3.20: Optimization convergence histories for CRM wing optimization - 5 streamwise control points per patch

weaken or eliminate shock waves, in order to reduce wave drag. The airfoil shapes show that the leading edge is becoming pointed near the wing tip. This feature is undesirable structurally and aerodynamically at lower angles of attack, and can be avoided by using geometric constraints or performing a multipoint optimization with cases at lower angles of attack. 


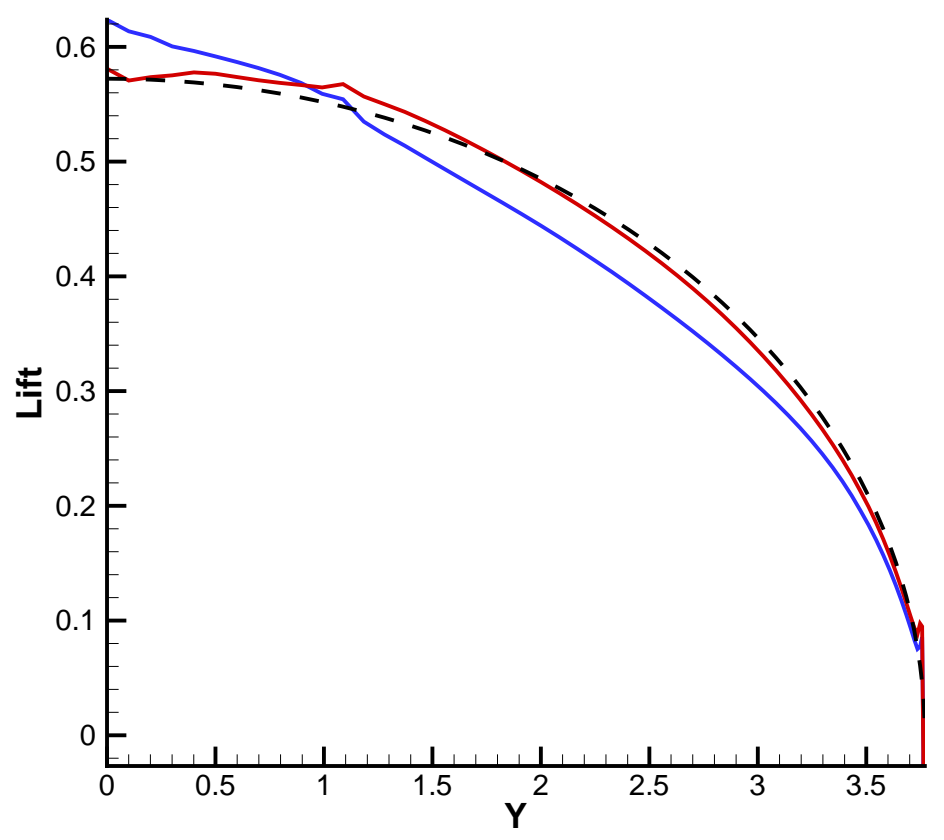

Figure 3.21: Spanwise lift distribution for CRM wing optimization with 5 streamwise and 7 spanwise control points per patch 


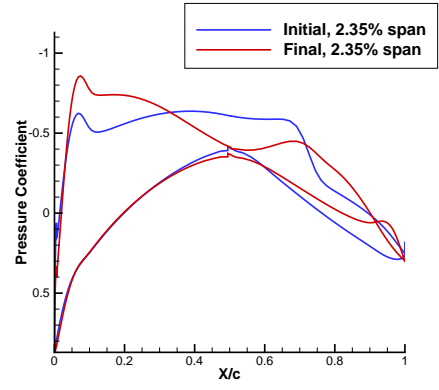

(a) $2.35 \%$ Span

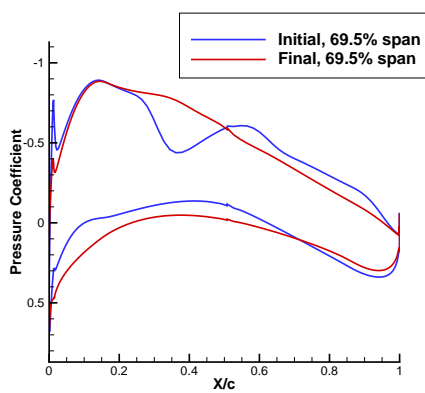

(d) $69.5 \%$ Span

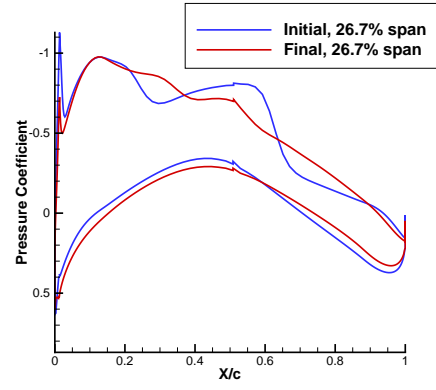

(b) $26.7 \%$ Span

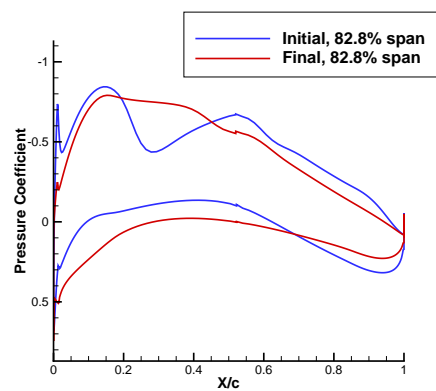

(e) $82.8 \%$ Span

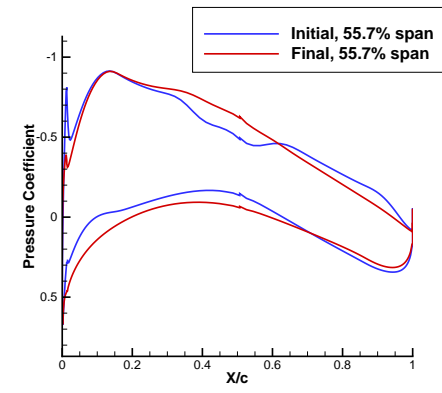

(c) $55.7 \%$ Span

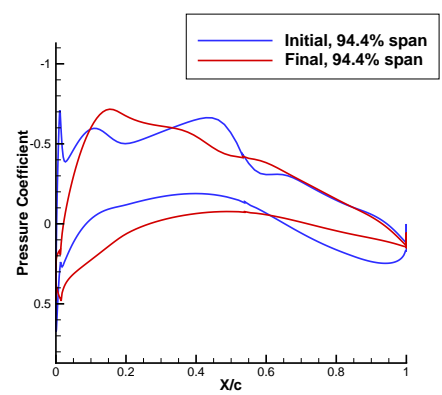

(f) $94.4 \% \mathrm{Span}$

Figure 3.22: Comparison of initial and final surface pressure coefficient distributions for CRM wing optimization with 5 streamwise and 7 spanwise control points per patch 


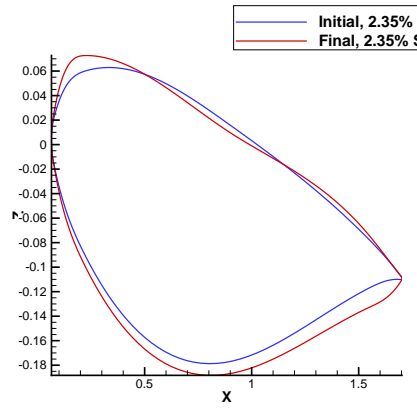

(a) $2.35 \%$ Span

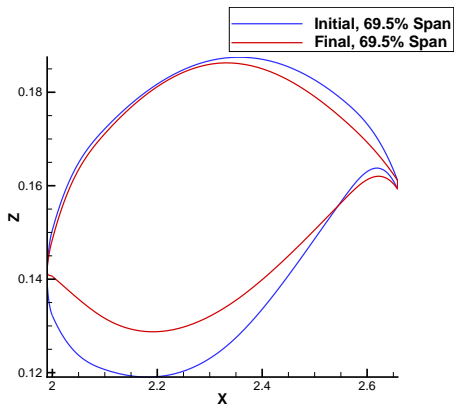

(d) $69.5 \% \mathrm{Span}$

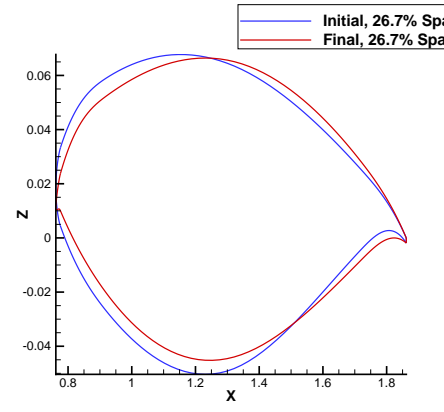

(b) $26.7 \%$ Span

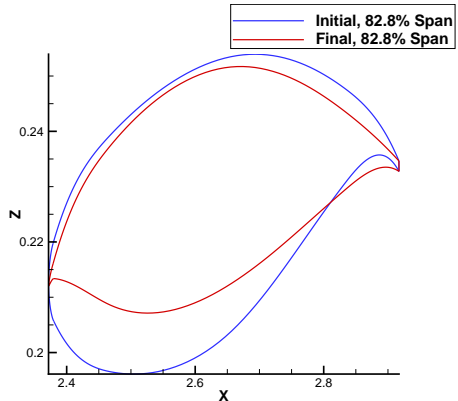

(e) $82.8 \% \mathrm{Span}$

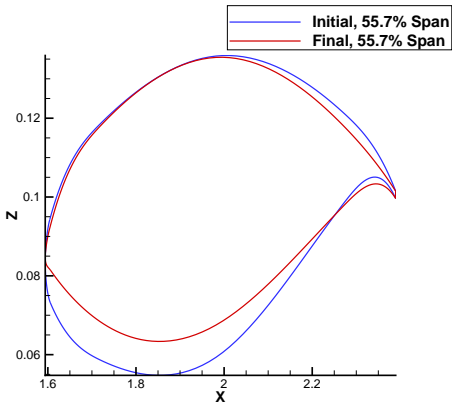

(c) $55.7 \%$ Span

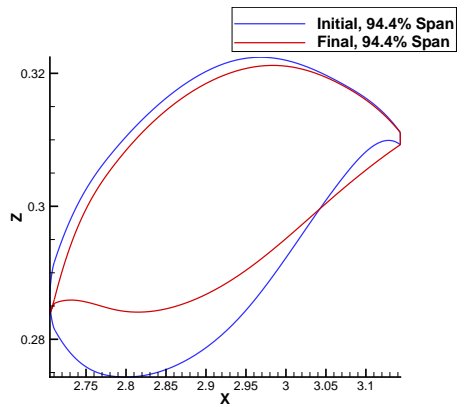

(f) $94.4 \% \mathrm{Span}$

Figure 3.23: Comparison of initial and final airfoil shapes for CRM wing optimization with 5 streamwise and 7 spanwise control points per patch 



\section{Chapter 4}

\section{Conclusions and Recommendations}

\subsection{Conclusions}

The objective of this thesis is to investigate the impact of the number of design variables on three standard aerodynamic shape optimization test cases. The goal is to provide insight into the impact of dimensionality on aerodynamic shape optimization. The three cases investigated are:

1. drag minimization of a symmetrical two-dimensional airfoil in transonic inviscid flow, subject to a thickness constraint that requires the airfoil to have thickness greater than or equal to the NACA 0012 airfoil;

2. lift-constrained drag minimization through optimizing the twist distribution of a rectangular wing with NACA 0012 sections in subsonic inviscid flow;

3. drag minimization through optimizing the twist distribution and section shapes of the CRM blunt trailing edge wing in transonic turbulent flow subject to lift and pitching moment constraints.

Due to the thickness constraint imposed for the first case, shock waves cannot be completely eliminated. The optimizer thickens the airfoil near the trailing edge and is able to weaken the shocks and move them aft. As the number of design variables increases, the general trend shows a decrease in the final drag coefficient. However, the decrease is not strictly monotonic, 
and as the number of design variables increases, the optimizer is not able to achieve significant reductions in optimality. As a result, the drag coefficient begins to increase. The lowest drag coefficient is obtained with 9 design variables, and is reduced by $91 \%$ to 42 drag counts. The results show that a finer parameterization is required to gain enough flexibility for the optimizer to reduce the drag significantly; however this comes at a cost to optimization convergence.

For the second case, the optimization algorithm is able to produce a known result, the elliptical spanwise lift distribution due to minimum induced drag for a planar wake. As the number of spanwise control points increases, the drag coefficient decreases and the span efficiency factor increases. However, the difference between the coarsest and finest parameterization is small. The number of spanwise control points has no effect on the convergence of the optimizer, with all cases able to converge to the required tolerance. The drag is reduced $3 \%$ and the span efficiency factor is increased $3 \%$ from the initial geometry.

The third case introduces optimization based on the RANS equations. In contrast to the first two cases, where most optimizations achieved the required optimality measure, the optimality is not well converged for any of the parameterizations examined. Nevertheless, the drag coefficient is consistently reduced. The number of streamwise and spanwise control points is varied from 5 to 9 in the streamwise direction and 5 to 7 in the spanwise direction, for a total of 15 different parameterizations. The drag coefficient decreases as the number of spanwise control points increases, and the lowest drag coefficient is seen with the coarsest streamwise parameterization (5 control points per patch) and the finest spanwise parameterization ( 7 control points per patch). The difference between the best and worst cases is 1.7 drag counts. For the best case, the optimizer is able to achieve a drag reduction of 20 counts, or $9 \%$, on the grid used for optimization.

The optimization algorithm is able to achieve significant drag reductions in all three cases studied. Valuable insight into the impact of dimensionality is gained, showing that increasing the number of control points used to parameterize the geometry can provide greater flexibility to the optimization algorithm. This flexibility can come at a cost to the convergence of the optimization algorithm, whether that be due to an inability to reduce the optimality significantly, the increased geometric flexibility challenging the mesh movement algorithm, or the unique aerodynamic shapes being unable to achieve converged flow solutions. 


\subsection{Recommendations}

For the first case, the number of design variables should be further increased beyond 11 to see what impact increased dimensionality has. Other researchers who examined this test case used as many as 64 control points (which would equate to 62 design variables). Many of the final solutions are not in the grid converged region, so further study of the grid convergence of the final solutions is required. For the second case, it would be preferable to analyze with a coarser parameterization. Due to the grid created, the rectangular wing has 16 surface patches, with each patch parameterized with 13 streamwise and 5 to 11 spanwise control points. This is a relatively fine geometry parameterization. Using fewer surface patches, or decoupling the number of design variables from the grid blocking, would allow a coarser parameterization to be used. The optimizer produced a twist distribution with drastic changes in the twist angle near the root, which is not desirable structurally. Imposing constraints to prevent this or performing aerostructural optimization is recommended. For the third case, a complete grid convergence study should be performed, particularly on the final optimized geometries.

The cases would benefit from analysis using free-form deformation (FFD), which would allow for a larger number of geometric control points to be used while maintaining a manageable number of design variables for the optimization algorithm. The first case would particularly benefit from this treatment, as it shows a degradation in optimizer performance as the geometry parameterization is refined. Chernukhin and Zingg [6] investigated the multimodality of several types of problems, including an airfoil, wing, and blended-wing-body. It would be interesting to investigate if any of the 3 cases show multimodality, and particularly if the number of design variables could impact multimodality. 



\section{References}

[1] O. Amoignon, J. Navratil, and J. Hradil. Study of parameterizations in the project CEDESA. In 52nd AIAA Aerospace Sciences Meeting, number AIAA-2014-0570, National Harbor, Maryland, January 2014.

[2] M. Andreoli, A. Janke, and J-A. Desideri. Free-form-deformation parameterization for multilevel 3D shape optimization in aerodynamics. Technical report, Institut National de Recherche en Informatique et en Automatique, 2003.

[3] F. Bisson, S. Nadarajah, and D. Shi-Dong. Adjoint-based aerodynamic optimization of benchmark problems. In 52nd AIAA Aerospace Sciences Meeting, number AIAA-20140412, National Harbor, Maryland, January 2014.

[4] H. P. Buckley and D. W. Zingg. On aerodynamic design through multipoint numerical optimization. In 49th AIAA Aerospace Sciences Meeting, number AIAA-2011-1244, Orlando, Florida, January 2011.

[5] G. Carrier, D. Destarac, A. Dumont, M. Meheut, I. Salah El Din, J. Peter, and S. Ben Khelil. Gradient-based aerodynamic optimization with the elsA software. In 52nd AIAA Aerospace Sciences Meeting, number AIAA-2014-0568, National Harbor, Maryland, January 2014.

[6] O. Chernukhin and D. W. Zingg. Multimodality and global optimization in aerodynamic design. AIAA Journal, 51(6):1342-1354, June 2013.

[7] Carl de Boor. A Practical Guide to Splines. Springer-Verlag Berlin and Heidelberg GmbH and Co., 1978. 
[8] P. E. Gill, W. Murray, and M. A. Saunders. SNOPT : An SQP algorithm for large-scale constrained optimization. SIAM Review, 47(1):99-131, 2005.

[9] X. Han and D. W. Zingg. An adaptive geometry parametrization for aerodynamic shape optimization. Journal of Optimization and Engineering, 15(1):69-91, March 2014.

[10] J. E. Hicken and D. W. Zingg. A parallel Newton-Krylov solver for the Euler equations discretized using simultaneous approximation terms. AIAA Journal, 46(11):2773-2786, November 2008.

[11] J. E. Hicken and D. W. Zingg. Aerodynamic optimization algorithm with integrated geometry parameterization and mesh movement. AIAA Journal, 48(2):400-413, February 2010 .

[12] J. E. Hicken and D. W. Zingg. Aerodynamic optimization algorithm with integrated geometry parameterization and mesh movement. AIAA Journal, 48(2):400-413, 2010.

[13] J. Hoschek. Intrinsic parametrization for approximation. Computer Aided Geometric Design, 5(1):27-31, June 1988.

[14] IATA. Vision 2050. Technical Report February, 2011.

[15] A. Jameson. Aerodynamic design via control theory. Journal of Scientific Computing, 3(3):233-260, 1988.

[16] N. Kumar, A. Diwakar, S. K. Attree, and S. Mittal. A method to carry out shape optimization with a large number of design variables. International Journal for Numerical Methods in Fluids, 71:1494-1508, August 2013.

[17] D. W. Levy, K. R. Laflin, E. N. Tinoco, J. C. Vassberg, M. Mani, B. Rider, C. L. Rumsey, R. A. Wahls, J. H. Morrison, O. P. Brodersen, S. Crippa, D. J. Mavriplis, and M. Murayama. Summary of data from the Fifth AIAA CFD Drag Prediction Workshop. In 51st AIAA Aerospace Sciences Meeting and Aerospace Exposition, number AIAA-2013-0046, Grapevine, Texas, United States, January 2013. 
[18] J. A. Meijerink and H. A. van der Vorst. An iterative solution method for linear systems of which the coefficient matrix is a symmetric m-matrix. Mathematics of Computation, 31(137):148-162, January 1977.

[19] L. Osusky. A numerical methodology for aerodynamic shape optimization in turbulent flow enabling large geometric variation. PhD thesis, University of Toronto, 2013.

[20] L. Osusky and D. W. Zingg. A Novel Aerodynamic Shape Optimization Approach for Three-Dimensional Turbulent Flows. In 50th AIAA Aerospace Sciences Meeting, number AIAA-2012-0058, Nashville, Tennessee, United States, January 2012.

[21] L. Osusky and D. W. Zingg. Application of an efficient Newton-Krylov algorithm for aerodynamic shape optimization based on the Reynolds-Averaged Navier-Stokes equations. In 21st AIAA Computational Fluid Dynamics Conference, number AIAA-2013-2584, San Diego, California, United States, June 2013.

[22] M. Osusky, J. E. Hicken, and D. W. Zingg. A parallel Newton-Krylov-Schur flow solver for the Navier-Stokes equations using the SBP-SAT approach. In 48th AIAA Aerospace Sciences Meeting and Aerospace Exposition, number AIAA-2010-116, Orlando, Florida, United States, January 2010.

[23] K. Ou, A. Jameson, and J. C. Vassberg. Airfoils supporting non-unique transonic solutions for unsteady viscous flows. In 7th AIAA Theoretical Fluid Mechanics Conference, number AIAA-2014-2927, June 2014.

[24] O. Pironneau. Optimal shape design for elliptic systems. Springer-Verlag, 1983.

[25] P. Thokala and J. R. R. A. Martins. Variable-complexity optimization applied to airfoil design. Engineering Optimization, 39(3):271-286, April 2007.

[26] A. H. Truong, C. A. Oldfield, and D. W. Zingg. Mesh movement for a discrete adjoint Newton-Krylov algorithm for aerodynamic optimization. AIAA Journal, 46(7):1695-1704, July 2008 .

[27] J. C. Vassberg, N. A. Harrison, D. L. Roma., and A. Jameson. A systematic study on the impact of dimensionality for a two-dimensional aerodynamic optimization model problem. 
In 29th AIAA Applied Aerodynamics Conference, number AIAA-2011-3176, pages 1-19, Honolulu, Hawaii, June 2011.

[28] J. C. Vassberg, E. N. Tinoco, M. Mani, B. Rider, T. Zickuhr, D. W. Levy, O. P. Brodersen, B. Eisfeld, S. Crippa, R. A. Wahls, J. H. Morrison, D. J. Mavriplis, and M. Murayama. Summary of the fourth AIAA CFD Drag Prediction Workshop. In 28th AIAA Applied Aerodynamics Conference, number AIAA-2010-4547, Chicago, Illinois, United States, June 2010.

[29] D. W. Zingg, M. Nemec, and T. H. Pulliam. A comparative evaluation of genetic and gradient-based algorithms applied to aerodynamic optimization. REMN, 48(2):103-126, February 2008. (invited paper in a special issue on adjoint-based shape design in Revue Europeenne de Mecanique Numerique - European Journal of Computational Mechanics). 